\title{
Dipole excitation of surface plasmon on a conducting sheet: finite element approximation and validation
}

\author{
Matthias Maier ${ }^{\mathrm{a}, 1}$, Dionisios Margetis ${ }^{\mathrm{b}, 2}$, Mitchell Luskin ${ }^{\mathrm{a}, 1, *}$ \\ ${ }^{a}$ School of Mathematics, University of Minnesota, Minneapolis, Minnesota 55455, USA. \\ ${ }^{b}$ Department of Mathematics, and Institute for Physical Science and Technology, and Center \\ for Scientific Computation and Mathematical Modeling, University of Maryland, College \\ Park, Maryland 20742, USA.
}

\begin{abstract}
We formulate and validate a finite element approach to the propagation of a slowly decaying electromagnetic wave, called surface plasmon-polariton, excited along a conducting sheet, e.g., a single-layer graphene sheet, by an electric Hertzian dipole. By using a suitably rescaled form of time-harmonic Maxwell's equations, we derive a variational formulation that enables a direct numerical treatment of the associated class of boundary value problems by appropriate curl-conforming finite elements. The conducting sheet is modeled as an idealized hypersurface with an effective electric conductivity. The requisite weak discontinuity for the tangential magnetic field across the hypersurface can be incorporated naturally into the variational formulation. We carry out numerical simulations for an infinite sheet with constant isotropic conductivity embedded in two spatial dimensions; and validate our numerics against the closed-form exact solution obtained by the Fourier transform in the tangential coordinate. Numerical aspects of our treatment such as an absorbing perfectly matched layer, as well as local refinement and a posteriori error control are discussed.
\end{abstract}

Keywords: Time-harmonic Maxwell's equations, finite element method, surface plasmon-polariton, weak discontinuity on hypersurface

2010 MSC: 65N30, 78M10, 78M30, 78A45

\section{Introduction}

The manipulation of the electronic structure of low-dimensional materials has recently been the subject of active research with applications in spintronics,

\footnotetext{
* Corresponding author

Email addresses: msmaier@umn.edu (Matthias Maier), dio@math.umd.edu (Dionisios Margetis), luskin@umn.edu (Mitchell Luskin)

${ }^{1}$ The first and third authors (MM and ML) were supported in part by ARO MURI Award W911NF-14-1-0247. The third author (ML) was also supported in part by the Radcliffe Institute for Advanced Study at Harvard University.

${ }^{2}$ This author's research was supported in part by NSF DMS-1412769.
}

Preprint submitted to Journal of Computational Physics

March 4, 2017

(C) 2017. This manuscript version is made available under the Elsevier user license http://www.elsevier.com/open-access/userlicense/1.0/ 
quantum information processing, and energy storage [1, 2, 3, 4, 5, 6, 7]. In particular, the electric conductivity of atomically thick materials such as graphene and black phosphorous yields an effective complex permittivity with a negative real part in the infrared spectrum [7, 8. This feature allows for the propagation of slowly decaying electromagnetic waves, called surface plasmons-polaritons (SPPs), that are confined near the material interface with wavelengths much shorter than the wavelength of the free-space radiation [9, 7]. These SPPs are promising ingredients in the design of ultrafast photonic circuits $[9]$.

Experimental efforts to generate SPPs focus on the requisite phase matching between waves sustained in free space and the material of interest [10]. This matching is enabled by the excitation of sufficiently large wave numbers tangential to the associated interface. A technique to achieve this goal is to place a resonant antenna on a graphene sheet [11, 12. The prediction of the resulting waves relies on solving an intricate boundary value problem for the time-harmonic Maxwell equations.

Our purpose with this paper is three-fold. First, we aim to develop a general variational framework for the numerical treatment of electromagnetic-wave propagation in conducting materials modeled as hypersurfaces in a Euclidean space of arbitrary dimension. These hypersurfaces are irradiated by fields produced by external, compactly supported current-carrying sources operating at a fixed, yet arbitrary, frequency. Second, we validate this framework by comparison of finite element numerical simulations to an exact solution of Maxwell's equations in the simplified case when the conducting sheet is infinite and embedded in a two-dimensional (2D) space in the presence of a Hertzian electric dipole directed vertically to the sheet. Third, we demonstrate that our numerical approach is capable of efficiently resolving SPP structures. To this end, goal-oriented adaptivity for local mesh refinement and a perfectly matched layer are tailored to the fine structure of SPPs.

This work has been broadly motivated by the growing urge to engineer microscopic details of low-dimensional conducting materials in order to establish desired optical properties at larger scales [9]. A key objective is to elucidate how the electric conductivity of the material affects characteristics of electromagnetic wave propagation [13]. There is a compelling need for controllable numerical schemes which, placed on firm mathematical grounds, can reliably describe the SPPs in a variety of geometries. Our paper offers a systematic approach to solving this problem. In particular:

- We formulate a variational framework suitable for the finite element treatment of electromagnetic wave propagation along conducting sheets embedded in spaces of arbitrary dimensions in the presence of external sources (Section 2.3).

- We discretize our variational formulation by use of appropriate curlconforming Nédélec-elements. This scheme is implemented in a modern $\mathrm{C}++$ framework [14] and accounts for the fine scale of the SPP as well as for the requisite radiation condition at infinity via a perfectly matched 
layer (PML) (Section 4). Our approach does not require regularization of the conducting sheet.

- We validate our numerical treatment by comparison of numerics to a tractable exact solution in the simplified case with a vertical electric dipole radiating over an infinite, planar conducting sheet; for convenience, we consider an ambient 2D Euclidean space. In particular, we numerically single out the SPP; and also compare it to the slowly-varying radiation field (see Sections 3 and 5 .

The extensive literature in plasmonics attests to the rich variety of computational methods and tools; see, e.g., [15, 16]. For example, an approach is to model graphene as a region with finite thickness (as opposed to a boundary) [17. In the present work, we demonstrate the ability of curl-conforming Nédélec-elements to accurately capture the fine scale of the SPPs, by replacing the conducting sheet with a set of boundary conditions on a hypersurface.

Note that our setting and objectives in this work, focusing on the propagation of SPPs along low-dimensional conducting materials, are distinctly different from the modeling and computation of the interaction of plasmonic nanoparticles with electromagnetic fields [18, 19. Our focus on the computation of SPPs along low-dimensional conducting materials and its validation against recently derived analytic solutions [20] is also distinct from the more classical study of surface plasmons on bulk materials 21]. However, our formulation and general approach is also applicable in this setting.

Throughout the paper, we assume that the reader is familiar with the fundamentals of classical electromagnetic wave theory; for extensive and comprehensive treatments of this subject, see, e.g., 22, 23, 24].

\subsection{Motivation: Surface plasmonics}

Atomically thick conducting materials such as graphene, black phosphorus, and van der Waals heterostructures have been the focus of intensive studies [5, 9]. The dispersion relations of these structures for electromagnetic wave propagation have novel features. The implications of this dispersion at the infrared spectrum is a theme of essence in surface plasmonics [25, 7]. Specifically, in the terahertz frequency range, the effective electric conductivity, $\sigma^{\Sigma}$, emerging from the coordinated motion of quasi-free electrons, can have an appreciable imaginary part. Furthermore, it has been predicted via numerical simulations that the decoration of graphene by chains of organic molecules may result in a dramatic alteration of $\sigma^{\Sigma}$ [13, 26]. This prediction paves the way to unconventional means of controlling electronic transport.

From the viewpoint of Maxwell's equations, the effective dielectric permittivity of a conducting sheet may have a negative real part. The resulting metamaterial has optical properties different from those of a conventional conductor [7]. In particular, electromagnetic waves of transverse-magnetic (TM) polarization possibly propagating through the atomically thick material are characterized by a dispersion relation that allows for transmitted wave numbers much larger 
than the free-space wave number, $k$. For an isotropic and homogeneous ambient space, with wave number $k=\omega \sqrt{\tilde{\varepsilon} \mu}$ and scalar and $\boldsymbol{x}$-independent $\mu$ and $\tilde{\varepsilon}$, and an isotropic and homogeneous conducting sheet, the condition $\left|\omega \mu \sigma^{\Sigma}\right| \ll|k|$ yields the simplified dispersion relation [25, 27, 21]

$$
\sqrt{k^{2}-k_{x}^{2}} \approx-\left(\frac{2 k}{\omega \mu \sigma^{\Sigma}}\right) k
$$

for TM waves; $k_{x}$ denotes the wave number tangential to the material interface. Hence, if $k$ is positive, (1) has an admissible solution, $k_{x}$, provided $\operatorname{Im} \sigma^{\Sigma}>0$ under an assumed $e^{-i \omega t}$ time dependence. Note that $\left|k_{x}\right| \gg|k|$.

The excitation of SPPs on a homogeneous conducting sheet cannot be achieved by direct illumination of the material by an incident plane wave. There is intrinsic need for phase matching between the waves propagating in different materials, e.g., air and conducting sheet [10, 25]. An indirect means of establishing this matching is to add metal contacts to the interface [28]. More generally, it is plausible to prescribe current-carrying sources of compact support that optimize attributes of the SPP by variation of the frequency or size of source or the esheet conductivity $13,12,11$.

\subsection{Our approach}

A jump condition created by an electric conductivity on an interface is a key ingredient in the modeling of thin conducting materials such as graphene, black phosphorus, and a variety of heterostructures resulting from stacking a few distinct crystalline sheets on top of each other. One of our tasks with this work is to construct a variational formulation, well-suited for the finite element method, that naturally incorporates such a jump condition in the presence of external sources.

The variational formulation is implemented by utilizing an appropriate curlconforming finite element space that only enforces the continuity of the tangential components across elements. The use of higher-order conforming elements is well suited for the numerical problem at hand. The weak discontinuity across the interface can be aligned with the triangulation and the regularity of the solution away from the interface leads to high convergence rates. For overcoming the two-scale character with much finer SPP structures close to the interface, an adaptive, local refinement strategy based on a posteriori error estimates is used. The a posteriori error estimates are computed by solving an adjoint problem (dual weighted residual method) 29] and lead to optimally refined meshes.

Notably, our approach does not require the regularization of the conducting sheet by a layer with artificial thickness. Instead, the sheet can be directly approximated as a lower-dimensional interface. Further, we treat the full scattering problem with an incident wave generated by a Hertzian dipole source instead of merely solving the associated eigenvalue problem for the SPP.

For validation of our treatment, the finite element computations stemming from our approach are compared to the exact solution of Maxwell's equations for a vertical electric dipole over an isotropic and homogeneous conducting sheet 
in 2D. In this case, all field components are expressed via 1D Fourier integrals and, thus, are amenable to accurate numerical integration. By this formalism, the SPP is defined as the contribution from a simple pole in the Fourier domain. This contribution is to be contrasted to the slowly-varying radiation field. Our numerics indicate that the SPP dominates the scattered field at distances of the order of the free-space wavelength from the dipole source, in agreement with analytical estimates from the exact solution.

\subsection{Related work}

Electromagnetic wave propagation along boundaries, especially the boundary separating air and earth or sea, has been the subject of studies for over a century. A review can be found in [22].

This insight is valuable yet insufficient for plasmonic applications related to low-dimensional materials. It is compelling to consider implications of the metamaterial character of atomically thick conducting sheets in the terahertz frequency range [10. In particular, in the presence of an electric Hertzian dipole source, boundary condition (3) with $\operatorname{Im} \sigma^{\Sigma}>0$ can result in a SPP [10], to be contrasted to surface waves in radio-frequencies which have wave numbers nearly equal to the free-space one [22].

In the last few decades, several groups have been studying implications of surface plasmonics; for a (definitely non-exhaustive) sample of related works, see [10, 27, 30, 12, 20, 31, 21, 25, 28. For instance, in [10] the authors review macroscopic properties of the electric conductivity of graphene, derive dispersion relations for electromagnetic plane waves in inhomogeneous structures, and discuss methods for exciting SPPs; see also the integral-equation approach in [28]. On the other hand, the problem of a radiating dipole source near a graphene sheet is semi-analytically addressed in [27, 30. In the same vein, in [31] the authors numerically study the field produced by dipoles near a graphene sheet, recognizing a region where the scattered field may be significant. Most recently, two of us derived closed-form analytical expressions for the electromagnetic field when the dipole source and observation point lie on the sheet [20].

In the aforementioned works, the rigorous numerical treatment of Maxwell's equations is not of primary concern. Our work here aims to build a framework that places the finite element treatment of a variational boundary value problem on firmer mathematical grounds. This opens up the possibility of numerically studying SPPs in experimentally accessible geometries in an error-controllable fashion. The finite element treatment of Maxwell's equations is a well-established area of research 32, 33, 34, 35, 36. In particular, our work is related to an adaptive finite element framework for Maxwell's equations [32, 33] that uses a residual-based a posteriori error estimator for adaptive local refinement of a triangular mesh. Further, we point out that the well-posedness of our variational approach (see Section 2 is closely connected to the question of well-posedness of time-harmonic Maxwell's equations with sign-changing dielectric permittivity 37. The reason for this connection is that the jump condition for a field component across a boundary with a complex-valued conductivity (that we 
use to model graphene) can be understood as the zero-thickness limit of a bulk graphene region with a negative dielectric permittivity.

\subsection{Open problems}

Our work here focuses on the development of a reasonably general variational framework. We validate this formulation by comparison of the ensuing finite element numerical computations to the exact solution of Maxwell's equations in a relatively simple yet nontrivial geometry in $2 \mathrm{D}$. We deem these tasks as necessary first steps in establishing the proposed numerical framework; these steps should precede applications to more complicated cases of physical interest.

Therefore, our work admits several extensions and leaves a few pending issues, from the viewpoints of both analysis and applications. For example, we have not made attempts to fully characterize error estimates following from our treatment. The subtleties related to the possible spatial variation or anisotropy of surface conductivity, $\sigma^{\Sigma}$, lie beyond our present scope. The numerics for a current-carrying source over a conducting film in 3D [27, 20] have not been carried out, because of the expensive computations involved. The more elaborate yet experimentally accessible case with a receiving antenna lying on the material interface [11, where the current distribution on the source forms part of the solution, is a promising topic of near-future investigation.

\subsection{Outline of paper}

The remainder of our paper is organized as follows. In Section 2 we provide the desired variational characterization for boundary value problem (2)-(4). Section 3 focuses on the derivation of an exact solution for (2) 4 in $2 \mathrm{D}$, assuming that the external current-carrying source is a vertical electric dipole and the conducting sheet is homogeneous and isotropic. In Section 4 we describe the discretization of our variational formulation in the context of finite elements; in particular, we discuss the error control by our treatment (Section 4.2). Section 5 present computational results stemming from our approach for an infinite conducting sheet in 2D, along with comparisons with an exact solution. Finally, Section 6 concludes our paper with a summary of our results and an outlook.

\section{Variational formulation}

In this section, we derive a variational formulation for the time-harmonic Maxwell equations with an interface jump condition. We introduce a slightly modified rescaling of the associated equations to dimensionless forms that are best suited for the numerical observation of the SPP in our treatment. The interface jump condition (3) enters the variational formulation in the form of a weak discontinuity (with the second jump-condition for $\boldsymbol{E}$ being naturally encoded in the ansatz space). 


\subsection{Preliminaries: Boundary value problem}

Next, we formulate the corresponding boundary value problem for the conducting sheet, emphasizing the discontinuity of the magnetic field across the sheet. The starting point of our analysis is the strong form of Maxwell's equations for the time-harmonic electromagnetic field, $(\mathcal{E}(\boldsymbol{x}, t), \mathcal{B}(\boldsymbol{x}, t))=\operatorname{Re}\left\{e^{-i \omega t}(\boldsymbol{E}(\boldsymbol{x}), \boldsymbol{B}(\boldsymbol{x}))\right\}$, viz., [24]

$$
\left\{\begin{aligned}
-i \omega \boldsymbol{B}+\nabla \times \boldsymbol{E} & =-\boldsymbol{M}_{a}, \\
\nabla \cdot \boldsymbol{B} & =\frac{1}{i \omega} \nabla \cdot \boldsymbol{M}_{a}, \\
i \omega \tilde{\varepsilon} \boldsymbol{E}+\nabla \times\left(\mu^{-1} \boldsymbol{B}\right) & =\boldsymbol{J}_{a}, \\
\nabla \cdot(\tilde{\varepsilon} \boldsymbol{E}) & =\frac{1}{i \omega} \nabla \cdot \boldsymbol{J}_{a} .
\end{aligned}\right.
$$

A few comments on (2) are in order. The (constant) parameter $\omega$ is the temporal angular frequency $(\omega>0)$. We assume that all material parameters are time independent; furthermore, the time-independent, externally applied electric- and magnetic-current densities, $\boldsymbol{J}_{a}(\boldsymbol{x})$ and $\boldsymbol{M}_{a}(\boldsymbol{x})$, respectively, arise from the time-harmonic densities $\mathcal{J}_{a}(\boldsymbol{x}, t)=\operatorname{Re}\left\{e^{-i \omega t} \boldsymbol{J}_{a}(\boldsymbol{x})\right\}$ and $\mathcal{M}_{a}(\boldsymbol{x}, t)=\operatorname{Re}\left\{e^{-i \omega t} \boldsymbol{M}_{a}(\boldsymbol{x})\right\}$. The second-rank tensors $\mu(\boldsymbol{x})$ and $\tilde{\varepsilon}(\boldsymbol{x})$ represent the effective magnetic permeability and complex permittivity of the corresponding medium; the latter is $\tilde{\varepsilon}(\boldsymbol{x})=\varepsilon(\boldsymbol{x})+i \sigma(\boldsymbol{x}) / \omega$, where $\varepsilon(\boldsymbol{x})$ and $\sigma(\boldsymbol{x})$ are the (second-rank tensorial) dielectric permittivity and conductivity. We assume that $(\boldsymbol{E}, \boldsymbol{B}),\left(\boldsymbol{J}_{a}, \boldsymbol{M}_{a}\right)$ and $(\tilde{\varepsilon}, \mu)$ in (2) are $\boldsymbol{x}$ dependent with some (weak) regularity of the fields to ensure unique solvability, as discussed in Section 2

Equations (2), interpreted in the strong sense, hold in appropriate unbounded regions of the $n$-dimensional Euclidean space, $\mathbb{R}^{n}(n=2,3)$, excluding the set of points comprising the conducting sheet. We now turn our attention to the requisite boundary conditions along the sheet. This is modeled as an idealized, oriented hypersurface $\Sigma, \Sigma \subset \mathbb{R}^{n}$, with unit normal $\boldsymbol{\nu}$ and effective surface conductivity $\sigma^{\Sigma}(\boldsymbol{x})[10,27,30$. This consideration amounts to a jump condition in the tangential component of the magnetic field while the tangential electric field is continuous, viz., [10]

$$
\left\{\begin{array}{c}
\boldsymbol{\nu} \times\left.\left\{\left(\mu^{-1} \boldsymbol{B}\right)^{+}-\left(\mu^{-1} \boldsymbol{B}\right)^{-}\right\}\right|_{\Sigma}=\left.\sigma^{\Sigma}(\boldsymbol{x})\{(\boldsymbol{\nu} \times \boldsymbol{E}) \times \boldsymbol{\nu}\}\right|_{\Sigma}, \\
\boldsymbol{\nu} \times\left.\left\{\boldsymbol{E}^{+}-\boldsymbol{E}^{-}\right\}\right|_{\Sigma}=0
\end{array}\right.
$$

where $\mathcal{F}^{ \pm}(\mathcal{F}=\boldsymbol{E}, \boldsymbol{B})$ is the restriction of the vector-valued solution to either side $( \pm)$ of the hypersurface. The surface conductivity, $\sigma^{\Sigma}(\boldsymbol{x})$, is in principle a second-rank tensor and is responsible for the creation of the SPP under the appropriate source $\left(\boldsymbol{J}_{a}, \boldsymbol{M}_{a}\right)$ [10; see section 1.1. At the terahertz frequency range in doped graphene, for example, it is possible that the jump in the tangential component of the magnetic field is small compared to the magnitude of the field itself [10]. For the appropriate polarization and imaginary part of $\sigma^{\Sigma}$, this feature may yield a surface wave, the SPP, with a wavelength of the order of a few microns, much smaller than the free-space wavelength [10, 27, 20]. 
In addition, the electromagnetic field $(\boldsymbol{E}, \boldsymbol{B})$ must satisfy the Silver-Müller radiation condition, an extension of the Sommerfeld radiation condition, if the ambient (unbounded) medium is isotropic [23]. This amounts to the requirement that $\mathcal{F}(\mathcal{F}=\boldsymbol{E}, \boldsymbol{B})$ approach a spherical wave uniformly in the radial direction as $|\boldsymbol{x}| \rightarrow \infty$ for points at infinity and away from the conducting sheet. We need to impose

$$
\lim _{|\boldsymbol{x}| \rightarrow \infty}\left\{\boldsymbol{B} \times \boldsymbol{x}-c^{-1}|\boldsymbol{x}| \boldsymbol{E}\right\}=0, \lim _{|\boldsymbol{x}| \rightarrow \infty}\{\boldsymbol{E} \times \boldsymbol{x}+c|\boldsymbol{x}| \boldsymbol{B}\}=0, x \notin \Sigma ;
$$

$c$ is the speed of light in the respective medium. In the formulation of our numerical scheme, we avoid making explicit use of condition (4) by using appropriate boundary conditions together with a PML, which eliminates reflection from infinity.

\subsection{Rescaling}

Loosely following Colton and Kress [38, as well as Monk [34, we introduce a rescaling for the time-harmonic Maxwell equations (2). The key differences of our formulation from the above treatments [38, 34] are:

- The additional rescaling of every length scale in our problem by the freespace wavelength $2 \pi k_{0}^{-1}:=2 \pi\left(\omega \sqrt{\varepsilon_{0} \mu_{0}}\right)^{-1}$, where $\epsilon_{0}$ and $\mu_{0}$ denote the vacuum dielectric permittivity and magnetic permeability, respectively. This rescaling recognizes that the typical length scale of the SPP is one to two orders of magnitude smaller than the corresponding free-space wavelength [10; consequently, $1 / k_{0}$ is the appropriate macroscopic length scale.

- The rescaling of $\boldsymbol{E}, \boldsymbol{B}, \boldsymbol{J}_{a}$, and $\boldsymbol{M}_{a}$ by a typical electric current strength, $J_{0}$. In our case, $J_{0}$ is the strength of the prescribed dipole source at location $\boldsymbol{a}$ in the $\boldsymbol{e}_{i}$ direction in Cartesian coordinates:

$$
\boldsymbol{J}_{a}=J_{0} \boldsymbol{e}_{i} \delta(\boldsymbol{x}-\boldsymbol{a}) .
$$

Accordingly, we rescale $\mu$ and $\tilde{\varepsilon}$ by $\mu_{0}$ and $\varepsilon_{0}$, respectively; cf. (2):

$$
\mu \quad \longrightarrow \quad \mu_{r}=\frac{1}{\mu_{0}} \mu, \quad \tilde{\varepsilon} \quad \longrightarrow \quad \tilde{\varepsilon}_{r}=\frac{1}{\varepsilon_{0}} \tilde{\varepsilon} .
$$

Furthermore, by use of the free-space wave number, $k_{0}=\omega \sqrt{\varepsilon_{0} \mu_{0}}$, and the dipole strength, $J_{0}$, the rescaling of the vector fields and coordinates is carried out:

$$
\begin{array}{rlrl}
\boldsymbol{x} & \longrightarrow \hat{\boldsymbol{x}}=k_{0} \boldsymbol{x}, & \nabla & \longrightarrow \hat{\nabla}=\frac{1}{k_{0}} \nabla, \\
\boldsymbol{J}_{a} \longrightarrow \hat{\boldsymbol{J}}_{a}=\frac{1}{J_{0}} \boldsymbol{J}_{a}, & \boldsymbol{M}_{a} \longrightarrow \hat{\boldsymbol{M}}_{a}=\frac{k_{0}}{\omega \mu_{0} J_{0}} \boldsymbol{M}_{a}, \\
\boldsymbol{E} \longrightarrow \hat{\boldsymbol{E}}=\frac{k_{0}^{2}}{\omega \mu_{0} J_{0}} \boldsymbol{E}, & \boldsymbol{B} \longrightarrow \hat{\boldsymbol{B}}=\frac{k_{0}}{J_{0}} \mu^{-1} \boldsymbol{B} .
\end{array}
$$




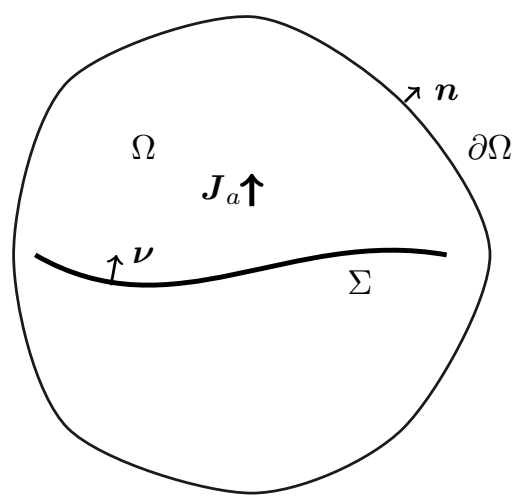

Figure 1: Schematic of the computational domain, $\Omega$, with boundary $\partial \Omega$ and outer normal $\boldsymbol{n}$. An electric Hertzian dipole, $\boldsymbol{J}_{a}$, is situated above a prescribed hypersurface, $\Sigma$.

In addition, the interface conductivity is rescaled as follows:

$$
\sigma^{\Sigma} \quad \longrightarrow \quad \sigma_{r}^{\Sigma}=\sqrt{\frac{\mu_{0}}{\varepsilon_{0}}} \sigma^{\Sigma}
$$

Finally, rescaling time-harmonic Maxwell's equations (2) results in the following system:

$$
\left\{\begin{aligned}
-i \mu_{r} \hat{\boldsymbol{B}}+\hat{\nabla} \times \hat{\boldsymbol{E}} & =-\hat{\boldsymbol{M}}_{a}, \\
\hat{\nabla} \cdot\left(\mu_{r} \hat{\boldsymbol{B}}\right) & =\frac{1}{i} \hat{\nabla} \cdot \hat{\boldsymbol{M}}_{a} \\
-i \tilde{\varepsilon}_{r} \hat{\boldsymbol{E}}-\hat{\nabla} \times \hat{\boldsymbol{B}} & =-\hat{\boldsymbol{J}}_{a} . \\
\hat{\nabla} \cdot\left(\tilde{\varepsilon}_{r} \hat{\boldsymbol{E}}\right) & =\frac{1}{i} \nabla \cdot \hat{\boldsymbol{J}}_{a} .
\end{aligned}\right.
$$

To lighten the notation, the hat $\left({ }^{\wedge}\right)$ on top of a rescaled quantity will be omitted in the remainder of this paper. (This simplification avoids confusion of the rescaled quantities with the Fourier transforms of fields invoked in Section 3 ).

\subsection{Variational statement}

Let $\Omega \subset \mathbb{R}^{n}(n=2,3)$ be a simply connected and bounded domain with Lipschitz-continuous and piecewise smooth boundary, $\partial \Omega$. Further, let $\Sigma$ be an oriented, Lipschitz-continuous, piecewise smooth hypersurface. Fix a normal field $\boldsymbol{\nu}$ on $\Sigma$ and let $\boldsymbol{n}$ denote the outer normal vector on $\partial \Omega$; see Figure 1 . Substituting $\boldsymbol{B}$ from the first equation of $(10)$ into the third equation yields

$$
\nabla \times\left(\mu_{r}^{-1} \nabla \times \boldsymbol{E}\right)-\tilde{\varepsilon}_{r} \boldsymbol{E}=i \boldsymbol{J}_{a}-\nabla \times\left(\mu_{r}^{-1} \boldsymbol{M}_{a}\right) .
$$


Multiplying (11) by the complex conjugate, $\bar{\varphi}$, of a smooth test function $\varphi$ and integrating by parts in $\Omega \backslash \Sigma$ lead to

$$
\begin{aligned}
\int_{\Omega}\left(\mu_{r}^{-1} \nabla \times \boldsymbol{E}\right) & \cdot(\nabla \times \overline{\boldsymbol{\varphi}}) \mathrm{d} x-\int_{\Omega} \tilde{\varepsilon}_{r} \boldsymbol{E} \cdot \overline{\boldsymbol{\varphi}} \mathrm{d} x \\
- & \int_{\Sigma}\left[\boldsymbol{\nu} \times\left(\mu_{r}^{-1} \nabla \times \boldsymbol{E}+\mu_{r}^{-1} \boldsymbol{M}_{a}\right)\right]_{\Sigma} \cdot \overline{\boldsymbol{\varphi}}_{T} \mathrm{~d} o_{x} \\
+ & \int_{\partial \Omega}\left(\boldsymbol{\nu} \times\left(\mu_{r}^{-1} \nabla \times \boldsymbol{E}+\mu_{r}^{-1} \boldsymbol{M}_{a}\right)\right) \cdot \overline{\boldsymbol{\varphi}}_{T} \mathrm{~d} o_{x} \\
& =i \int_{\Omega} \boldsymbol{J}_{a} \cdot \overline{\boldsymbol{\varphi}} \mathrm{d} x-\int_{\Omega} \mu_{r}^{-1} \boldsymbol{M}_{a} \cdot \nabla \times \overline{\boldsymbol{\varphi}} \mathrm{d} x
\end{aligned}
$$

where the subscript $T$ above denotes the tangential part of the respective vector, $\mathcal{F}_{T}=(\boldsymbol{\nu} \times \mathcal{F}) \times \boldsymbol{\nu}$, and $[.]_{\Sigma}$ denotes the jump over $\Sigma$ with respect to $\boldsymbol{\nu}$, viz.,

$$
[\mathcal{F}]_{\Sigma}(\boldsymbol{x}):=\lim _{s \searrow 0}(\mathcal{F}(\boldsymbol{x}+s \boldsymbol{\nu})-\mathcal{F}(\boldsymbol{x}-s \boldsymbol{\nu})) \quad(\boldsymbol{x} \in \Sigma) .
$$

For the computational domain $\Omega$, an absorbing boundary condition at $\partial \Omega$ is imposed:

$$
\boldsymbol{\nu} \times \boldsymbol{B}+\sqrt{\mu_{r}^{-1} \tilde{\varepsilon}_{r}} \boldsymbol{E}_{T}=0 \quad(\boldsymbol{x} \in \partial \Omega) .
$$

The last boundary condition is obtained by using a first-order approximation of the Silver-Müller radiation condition, equation (4), truncated at $\partial \Omega[39$. We will occasionally refer to (14) as the first-order absorbing boundary condition. We assume that $\mu_{r}^{-1}$ and $\tilde{\varepsilon}_{r}$ are scalar functions such that the square root in (14) is well defined. In our numerical computation, we combine the above absorbing boundary condition with a PML; see Section 5.2

An advantage of variational formulation 12 is that the jump condition over the conducting sheet can be expressed as a weak discontinuity. Rewriting jump condition (3) as well as absorbing boundary condition (14) in terms of $\boldsymbol{B}$ and $\boldsymbol{M}_{a}$ by utilizing the first equation of 10 yields

$$
\begin{aligned}
{\left[\boldsymbol{\nu} \times\left(\mu_{r}^{-1} \nabla \times \boldsymbol{E}+\mu_{r}^{-1} \boldsymbol{M}_{a}\right)\right]_{\Sigma} } & =i \sigma_{r}^{\Sigma} \boldsymbol{E}_{T} \quad \text { on } \Sigma, \\
\boldsymbol{\nu} \times\left(\mu_{r}^{-1} \nabla \times \boldsymbol{E}+\mu_{r}^{-1} \boldsymbol{M}_{a}\right) & =-i \sqrt{\mu_{r}^{-1} \tilde{\varepsilon}_{r}} \boldsymbol{E}_{T} \quad \text { on } \partial \Omega .
\end{aligned}
$$

The last two relations allow us to enforce the jump and boundary conditions weakly by simply substituting them into 12 . We summarize our main result below.

Proposition 1 (Variational formulation). In order to ensure unique solvability, we assume that $\sigma_{r}^{\Sigma} \in L^{\infty}(\Sigma)^{3 \times 3}$ is matrix-valued and symmetric, with semidefinite real and complex part. Further, let $\tilde{\varepsilon}_{r}$ be a smooth scalar function and $\mu_{r}^{-1}$ be a constant scalar such that

$$
-\operatorname{Im}\left(\tilde{\varepsilon}_{r}\right)=0 \text {, or } \operatorname{Im}\left(\tilde{\varepsilon}_{r}\right) \geq c>0 \text { in } \Omega,
$$


- $\sqrt{\mu_{r}^{-1} \tilde{\varepsilon}_{r}}$ is real-valued and strictly positive on $\partial \Omega$.

Define a Hilbert space (cf. 34, Th. 4.1])

$$
\boldsymbol{X}(\Omega)=\left\{\boldsymbol{\varphi} \in \boldsymbol{H}(\mathrm{curl} ; \Omega):\left.\boldsymbol{\varphi}_{T}\right|_{\Sigma} \in L^{2}(\Sigma)^{3},\left.\boldsymbol{\varphi}_{T}\right|_{\partial \Omega} \in L^{2}(\partial \Omega)^{3}\right\}
$$

equipped with the norm $\|\varphi\|_{\boldsymbol{X}}^{2}=\|\nabla \times \varphi\|^{2}+\left\|\varphi_{T}\right\|_{L^{2}(\Sigma)}^{2}+\left\|\varphi_{T}\right\|_{L^{2}(\Omega)}^{2}$. In the above, $\boldsymbol{H}($ curl; $\Omega)$ denotes the space of vector-valued, measurable and square integrable functions whose (distributive) curl admits a representation by a square integrable function.

The rescaled, weak formulation of (2) with jump condition (3) and absorbing boundary condition (14) can be stated as follows: Find $\boldsymbol{E} \in \boldsymbol{X}(\Omega)$, such that

$$
A(\boldsymbol{E}, \boldsymbol{\varphi})=F(\boldsymbol{\varphi})
$$

for all $\boldsymbol{\varphi} \in \boldsymbol{X}(\Omega)$. It admits a unique solution. The sesquilinear form and the right-hand side are given by

$$
\begin{aligned}
A(\boldsymbol{E}, \boldsymbol{\varphi}):= & \int_{\Omega}\left(\mu_{r}^{-1} \nabla \times \boldsymbol{E}\right) \cdot(\nabla \times \overline{\boldsymbol{\varphi}}) \mathrm{d} x-\int_{\Omega} \tilde{\varepsilon}_{r} \boldsymbol{E} \cdot \overline{\boldsymbol{\varphi}} \mathrm{d} x \\
& -i \int_{\Sigma}\left(\sigma_{r}^{\Sigma} \boldsymbol{E}_{T}\right) \cdot \overline{\boldsymbol{\varphi}}_{T} \mathrm{~d} o_{x}-i \int_{\partial \Omega} \sqrt{\mu_{r}^{-1} \tilde{\varepsilon}_{r}} \boldsymbol{E}_{T} \cdot \overline{\boldsymbol{\varphi}}_{T} \mathrm{~d} o_{x}, \\
F(\boldsymbol{\varphi}):= & i \int_{\Omega} \boldsymbol{J}_{a} \cdot \overline{\boldsymbol{\varphi}} \mathrm{d} x-\int_{\Omega} \mu_{r}^{-1} \boldsymbol{M}_{a} \cdot \nabla \times \overline{\boldsymbol{\varphi}} \mathrm{d} x .
\end{aligned}
$$

Proof. The existence result for time-harmonic Maxwells equations with an absorbing boundary condition [38, 40, 34, can be applied almost directly to problem (17); the additional interface integral in $A(\boldsymbol{E}, \boldsymbol{\varphi})$,

$$
-i \int_{\Sigma} \sigma_{r}^{\Sigma} \boldsymbol{E}_{T} \cdot \bar{\varphi}_{T} \mathrm{~d} o_{x}
$$

requires a careful discussion. For this we split the integral into two contributions,

$-i \int_{\Sigma} \sigma_{r}^{\Sigma} \boldsymbol{E}_{T} \cdot \overline{\boldsymbol{\varphi}}_{T} \mathrm{~d} o_{x}=-i \int_{\Sigma} \operatorname{Re}\left(\sigma_{r}^{\Sigma}\right) \boldsymbol{E}_{T} \cdot \overline{\boldsymbol{\varphi}}_{T} \mathrm{~d} o_{x}+\int_{\Sigma} \operatorname{Im}\left(\sigma_{r}^{\Sigma}\right) \boldsymbol{E}_{T} \cdot \overline{\boldsymbol{\varphi}}_{T} \mathrm{~d} o_{x}$.

The first term on the right-hand side of (21) can be treated similarly to the absorbing boundary condition on $\partial \Omega$ (cf. [34, Sec. 4.5]). For the second term in 21), involving $\operatorname{Im}\left(\sigma_{r}^{\Sigma}\right)$, it holds that

$$
\int_{\Sigma} \operatorname{Im}\left(\sigma_{r}^{\Sigma}\right) \boldsymbol{E}_{T} \cdot \overline{\boldsymbol{E}}_{T} \mathrm{~d} o_{x} \geq 0
$$

Thus, this term does not affect any proof based on showing coercivity of a modified sesquilinear form and using Fredholm's alternative on a compact perturbation. 
In order to prove uniqueness we follow the strategy in [34, Sec. 4.6]. First, test the homogeneous equation $A(\boldsymbol{e}, \boldsymbol{\varphi})=0$ with $\boldsymbol{e}$ itself and take the imaginary part,

$$
\int_{\Omega}\left(\operatorname{Im}\left(\tilde{\varepsilon}_{r}\right) \boldsymbol{e}\right) \cdot \overline{\boldsymbol{e}} \mathrm{d} x+\int_{\Sigma}\left(\operatorname{Im}\left(\sigma_{r}^{\Sigma}\right) \boldsymbol{e}_{T}\right) \cdot \overline{\boldsymbol{e}}_{T} \mathrm{~d} o_{x}+\int_{\partial \Omega} \sqrt{\mu_{r}^{-1} \tilde{\varepsilon}_{r}} \boldsymbol{e}_{T} \cdot \overline{\boldsymbol{e}}_{T} \mathrm{~d} o_{x}=0 .
$$

This immediately implies $\boldsymbol{e}_{T}=0$ on $\partial \Omega$ and $\Sigma$. The (nontrivial) conclusion $\boldsymbol{e}=0$ now follows verbatim by the proof of [34, Th.4.12].

The following remarks are in order.

Remark 1 (2D model of a conducting sheet). The $3 D$ problem description given by (17) readily translates into a corresponding problem in 2D: Assume that the interface $\Sigma$, the electric field $\boldsymbol{E}$, the permeability $\mu_{r}$, as well as the permittivity $\tilde{\varepsilon}_{r}$ and surface conductivity $\sigma_{r}^{\Sigma}$ are translation- and mirror-invariant in the $z$-direction. Thus, the term $\nabla \times \boldsymbol{E}$ and, consequently, the $\boldsymbol{B}$ field have nonzero components only in the $z$-direction. Hence, we can rewrite (17) with the $2 D$ curl

$$
\nabla \times \mathcal{F}:=\partial_{x} \mathcal{F}_{y}-\partial_{y} \mathcal{F}_{x}
$$

Remark 2 (TM polarization in 2D model). In the $2 D$ version of Maxwell's equations with a vertical electric dipole (see Section 3), the magnetic field, B, given by

$$
\boldsymbol{B}=\mu_{r}^{-1}\left(\boldsymbol{M}_{a}+\nabla \times \boldsymbol{E}\right), \quad \boldsymbol{M}_{a} \equiv 0,
$$

only has a z-component when viewed as a vector field in $\mathbb{R}^{3}$. Thus, this field is parallel to the hypersurface $\Sigma$ and orthogonal to the computational domain, $\Omega$ (which is part of the $x y$-plane). Consequently, the SPP in this $2 D$ setting, and in the corresponding numerical simulation, has the desired TM polarization [10].

\section{Exact solution for $2 \mathrm{D}$ infinite conducting sheet}

In this section, we derive an exact solution to the three-dimensional (3D) version of boundary value problem (2)-(4) in the case with a $2 \mathrm{D}$ vertical electric dipole radiating at frequency $\omega$ over an infinite conducting sheet embedded in an isotropic and homogeneous space of (scalar) magnetic permeability $\mu$; see Figure 2. For physical clarity, we invoke the vector-valued electromagnetic field without rescaling, unless we state otherwise.

The sheet separates the space into region $1(\{y>0\})$ with wave number $k_{1}$ and region $2(\{y<0\})$ with wave number $k_{2} ; k_{j}^{2}=\omega^{2} \tilde{\epsilon}_{j} \mu$ where $\tilde{\epsilon}_{j}$ is the complex permittivity $(j=1,2)$. We assume that the surface conductivity, $\sigma^{\Sigma}$, of the sheet is a scalar constant. Note that we assign different complex permittivities to each half-space (regions 1,2). In the end of this section, we set them equal. 


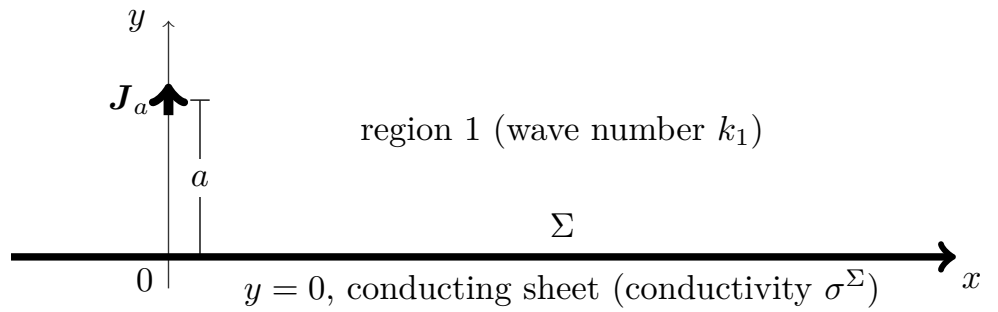

region 2 (wave number $k_{2}$ )

Figure 2: Schematic of a vertical electric dipole at distance $a$ from conducting sheet $\Sigma$ in 2D. The dipole has current density $\boldsymbol{J}_{a}=J_{0} \delta(\boldsymbol{x}-\boldsymbol{a}) e_{y}$, where $\boldsymbol{x}=x e_{x}+y e_{y}$ and $\boldsymbol{a}=a e_{y}$. The sheet lies in $y=0$, separates the space into region 1 (half space $\{y>0\}$ ) with wave number $k_{1}$ and region $2(\{y<0\})$ with wave number $k_{2}$, and has surface electric conductivity $\sigma^{\Sigma}$.

The dipole has current density $\boldsymbol{J}_{a}=J_{0} \delta(\boldsymbol{x}-\boldsymbol{a}) e_{y}$ where $\boldsymbol{x}=x e_{x}+y e_{y}$ and $\boldsymbol{a}=a e_{y} ; e_{s}$ is a unit Cartesian vector $(s=x, y)$. Now define the Fourier transform, $\widehat{\boldsymbol{F}}_{j}(\xi, y)$, of the vector-valued field $\boldsymbol{F}_{j}(x, y)(\boldsymbol{F}=\boldsymbol{E}, \boldsymbol{B})$ in region $j$ through the integral formula

$$
\boldsymbol{F}_{j}(x, y)=\frac{1}{2 \pi} \int_{\mathbb{R}} d \xi \widehat{\boldsymbol{F}}_{j}(\xi, y) e^{i \xi x} .
$$

The transformation of 3D Maxwell's equations (2) in region $j$ yields

$$
\begin{aligned}
& -i \xi \widehat{E}_{j y}+\frac{\partial \widehat{E}_{j x}}{\partial y}=-i \omega \widehat{B}_{j z}, \\
& -\frac{\partial \widehat{B}_{j z}}{\partial y}=\frac{i k_{j}^{2}}{\omega} \widehat{E}_{j x},-i \xi \widehat{B}_{j z}=-\frac{i k_{j}^{2}}{\omega} \widehat{E}_{j y}+\mu \delta(y-a),
\end{aligned}
$$

where we set $E_{j z} \equiv 0, B_{j x} \equiv 0$, and $B_{j y} \equiv 0$ by symmetry; $F_{j z}(F=E, B)$ is the component perpendicular to the $x y$-plane.

Equations 26) and (27) furnish the differential equation

$$
\frac{\partial^{2} \widehat{B}_{j z}}{\partial y^{2}}+\beta_{j}^{2} \widehat{B}_{j z}=-i \xi \mu \delta(y-a), \quad \beta_{j}^{2}:=k_{j}^{2}-\xi^{2},
$$

which has solution

$$
\widehat{B}_{j z}(\xi, y)= \begin{cases}C_{>} e^{i \beta_{1} y}-\frac{\xi \mu}{2 \beta_{1}} e^{i \beta_{1}|y-a|}, & \text { if } j=1(y>0) \\ C_{<} e^{-i \beta_{2} y}, & \text { if } j=2(y<0)\end{cases}
$$

This solution is consistent with radiation condition (4) provided

$$
\operatorname{Im} \beta_{j}(\xi)>0 \quad(j=1,2) .
$$


Furthermore, we apply conditions 3 in order to determine integration constants $C_{>}$and $C_{<}$. Specifically, we impose

$$
\left.\left(\widehat{B}_{1 z}-\widehat{B}_{2 z}\right)\right|_{y=0}=\left.\mu \sigma^{\Sigma} \widehat{E}_{1 x}\right|_{y=0},\left.\quad\left(\widehat{E}_{1 x}-\widehat{E}_{2 x}\right)\right|_{y=0}=0 .
$$

Accordingly, by (27) and (28) we find

$C_{>}=-\frac{\xi \mu}{2 \beta_{1}} \frac{k_{2}^{2} \beta_{1}-k_{1}^{2} \beta_{2}+\omega \mu \sigma^{\Sigma} \beta_{1} \beta_{2}}{k_{2}^{2} \beta_{1}+k_{1}^{2} \beta_{2}+\omega \mu \sigma^{\Sigma} \beta_{1} \beta_{2}} e^{i \beta_{1} a}, C_{<}=-\frac{\mu k_{2}^{2} \xi e^{i \beta_{1} a}}{k_{2}^{2} \beta_{1}+k_{1}^{2} \beta_{2}+\omega \mu \sigma^{\Sigma} \beta_{1} \beta_{2}}$.

We can now write down all field components in view of (25)-(28). In particular:

$$
\begin{aligned}
E_{1 x}(x, y)= & \frac{\omega \mu}{4 \pi k_{1}^{2}} \int_{-\infty}^{\infty} d \xi \xi\left[\frac{k_{2}^{2} \beta_{1}-k_{1}^{2} \beta_{2}+\omega \mu \sigma^{\Sigma} \beta_{1} \beta_{2}}{k_{2}^{2} \beta_{1}+k_{1}^{2} \beta_{2}+\omega \mu \sigma^{\Sigma} \beta_{1} \beta_{2}} e^{i \beta_{1}(y+a)}\right. \\
& \left.+\operatorname{sgn}(y-a) e^{i \beta_{1}|y-a|}\right] e^{i \xi x} \quad(y>0) ; \\
E_{2 x}(x, y)=- & \frac{\omega \mu}{2 \pi} \int_{-\infty}^{\infty} d \xi \xi \beta_{2} \frac{e^{i \beta_{1} a-i \beta_{2} y} e^{i \xi x}}{k_{2}^{2} \beta_{1}+k_{1}^{2} \beta_{2}+\omega \mu \sigma^{\Sigma} \beta_{1} \beta_{2}} \quad(y<0) .
\end{aligned}
$$

In the above, $\operatorname{sgn}(y)=1$ if $y>0$ and $\operatorname{sgn}(y)=-1$ if $y<0$.

Note that all field components have Fourier transforms defined in the $\xi$-plane with: (i) branch points at $\xi= \pm k_{j}(j=1,2)$; and (ii) simple poles at points where

$$
k_{2}^{2} \beta_{1}(\xi)+k_{1}^{2} \beta_{2}(\xi)+\omega \mu \sigma^{\Sigma} \beta_{1}(\xi) \beta_{2}(\xi)=0,
$$

under condition $(29$ which defines the appropriate branch of the multiple-valued $\beta_{j}(\xi)$. Equation (33) is the dispersion relation for the SPP [21, 20. In particular, if $k_{1}=k_{2}=k$ and $\left|\omega \mu \sigma^{\Sigma}\right| \ll|k|$, (33) reduces to (1) with $\xi=k_{x}$.

This observation motivates the following definition within the 2D model 20$]$.

Definition 1 (SPP in the 2D setting). For an infinite conducting sheet, the SPP is identified with the part of the electromagnetic field equal to the contribution to the Fourier integrals of the simple pole $\xi=k_{m}$, Imk $k_{m}>0$, that solves (33) under 29.

For the sake of completeness, we conclude this section by focusing on the case with $k_{1}=k_{2}=k$ (identical half-spaces). In particular, we provide explicit expressions for two distinct physical contributions to the $x$-component of the electric field on the sheet $(y=0)$, which is continuous across the sheet. After suitable rescaling of the variables and parameters (Section 2.2), by (32) the pole contribution to $E_{x}:=E_{1 x}(x, 0)=E_{2 x}(x, 0)$ takes the form

$$
E_{x, \mathrm{p}}=-2 i \frac{\mu_{r} \tilde{\varepsilon}_{r}}{\left(\sigma_{r}^{\Sigma}\right)^{2}} \exp \left[i k_{m, r} x-\left(2 i / \sigma_{r}^{\Sigma}\right) a_{r}\right],
$$


where $k_{m, r}=k_{m} / k_{0}$ and $a_{r}=k_{0} a$; cf. (33). Equation (34) expresses the SPP pertaining to the tangential electric field (see Definition 1).

A separate physical contribution expresses the radiation part of the scattered field, $\boldsymbol{E}^{\mathrm{sc}}=\boldsymbol{E}-\boldsymbol{E}^{\mathrm{pr}}$, where $\boldsymbol{E}^{\mathrm{pr}}$ is the (primary) dipole field in the absence of the sheet. To single out this contribution for the $x$-component, $E_{x}$, we choose to integrate in the $\xi$ (Fourier) variable around the branch cut emanating from the branch point $\xi=k$ after removal of the primary field component. The result reads

$$
\begin{gathered}
E_{x, \mathrm{bc}}^{\mathrm{sc}}=\frac{1}{4 \pi} \frac{1}{\sigma_{r}^{\Sigma}}\left\{\int_{0}^{1} \mathrm{~d} \xi \xi \sqrt{1-\xi^{2}} e^{i \sqrt{\mu_{r} \tilde{\varepsilon}_{r}} x \xi} \frac{1}{\xi^{2}+4 \frac{\mu_{r} \tilde{\varepsilon}_{r}}{\left(\sigma_{r}^{\Sigma}\right)^{2}}-1}\right. \\
\cdot\left(4 \sqrt{\mu_{r} \tilde{\varepsilon}_{r}} \cos \left(\sqrt{\mu_{r} \tilde{\varepsilon}_{r}} a \sqrt{1-\xi^{2}}\right)-2 i \sigma_{r}^{\Sigma} \sqrt{1-\xi^{2}} \sin \left(\sqrt{\mu_{r} \tilde{\varepsilon}_{r}} a \sqrt{1-\xi^{2}}\right)\right) \\
-\int_{0}^{\infty} \mathrm{d} \varsigma \varsigma \sqrt{1+\varsigma^{2}} e^{i \sqrt{\mu_{r} \tilde{\varepsilon}_{r}} x \varsigma} \frac{1}{\varsigma^{2}-4 \frac{\mu_{r} \tilde{\varepsilon}_{r}}{\left(\sigma_{r}^{\Sigma}\right)^{2}}-1} \\
\left.\cdot\left(4 \sqrt{\mu_{r} \tilde{\varepsilon}_{r}} \cos \left(\sqrt{\mu_{r} \tilde{\varepsilon}_{r}} a \sqrt{1+\varsigma^{2}}\right)-2 i \sigma_{r}^{\Sigma} \sqrt{1+\varsigma^{2}} \sin \left(\sqrt{\mu_{r} \tilde{\varepsilon}_{r}} a \sqrt{1+\varsigma^{2}}\right)\right)\right\} .
\end{gathered}
$$

Figure 3 shows a comparison of the two contributions, $E_{x, \mathrm{p}}$ and $E_{x, \mathrm{bc}}^{\mathrm{sc}}$, for a typical choice of parameters $\left(a=1.0, \sigma^{\Sigma}=2.00 \times 10^{-3}+0.200 i\right)$ by use of formulas (34) and (35) with $\mu_{r}=1$ and $\tilde{\varepsilon}_{r}=1$. We observe that the SSP (pole contribution) dominates in the (rescaled) range $10 \leq x \leq 25$ but is eventually dominated by the branch-cut contribution which has the (slower) algebraic decay.

\section{Numerics: Discretization scheme}

In this section, variational formulation (17) is discretized on a non-uniform quadrilateral mesh with higher-order, curl-conforming Nédélec elements [35. 36, 41. Such a conforming discretization is an ideal choice for the problem at hand. The interface with the (weak) discontinuity can be aligned with the mesh and away from it; and the regularity of the solution leads to high convergence rates. Key ingredient of our treatment is the use of an appropriately defined PML (Section 4.1), as well as local mesh refinement based on a posteriori error estimates (Section 4.2 ).

Let $X_{h}(\Omega) \subset X(\Omega)$ be a finite element subspace spanned by Nédélec elements. We will in particular use second-order Nédélec elements in the numerical computations. Then, under the assumption of a sufficiently refined initial mesh, the variational equation to find $E_{h} \in X_{h}(\Omega)$ such that

$$
A\left(\boldsymbol{E}_{h}, \boldsymbol{\varphi}\right)=F(\boldsymbol{\varphi}), \quad \forall \boldsymbol{\varphi} \in X_{h}(\Omega),
$$

is uniquely solvable [34, Section 7.2]. 


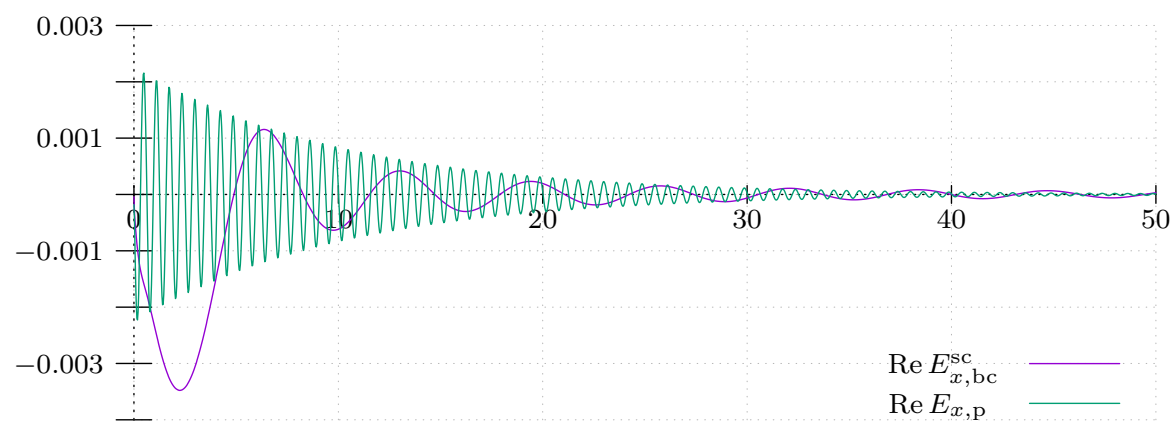

(a)

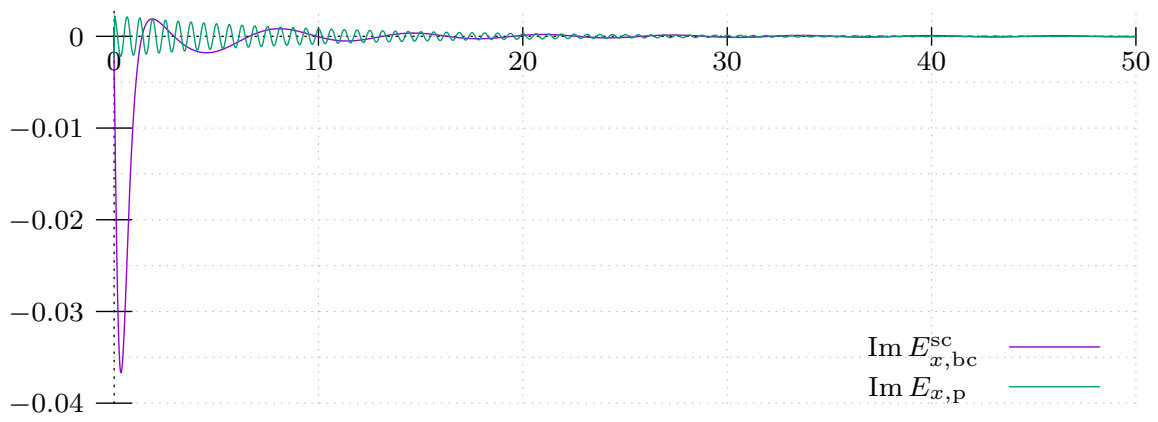

(b)

Figure 3: Real (a) and imaginary (b) part of branch-cut contribution 35 and SPP 34 for a dipole with (rescaled) elevation distance $a=1.0$ and SPP wave number $k_{m, r}=10.0+0.1 i$. The ambient space has $\tilde{\varepsilon}_{r}=1$ and $\mu_{r}=1$.

Remark 3. From an approximation theory point of view, we expect the convergence

$$
\left\|\boldsymbol{E}-\boldsymbol{E}_{h}\right\|_{X} \sim \mathcal{O}\left(h^{2}\right)=\mathcal{O}(\# d o f s)
$$

for second-order Nédélec elements and under the conditions of Proposition 1 , i. e., quadratic convergence in terms of a uniform refinement parameter $h$, or linear convergence in the number of degrees of freedom. This is evidenced by our numerical results presented in Section 5. We refrain from stating a rigorous convergence result at this point because the use of non-uniform, locally refined meshes with an additional approximation of a curved boundary significantly complicates the classical approximation theory (see 34, Section 7.3] and references therein).

The interface jump condition on the sheet introduces a pronounced two-scale character to the problem that needs special numerical treatment. Depending on the surface electric conductivity, $\sigma^{\Sigma}$, of the sheet, the observed SPP may have a rescaled wave number, $k_{m, r}$, that is one to two orders of magnitude higher 
than that produced by the dipole radiation in free space (which has rescaled wave number $k_{r}=1$ ). This fact has important consequences with respect to the refinement strategy and boundary conditions. In particular:

- First-order absorbing boundary conditions alone are in principle not well suited for conducting sheets sustaining SPPs. These boundary conditions lead to a significant suppression of the SPP amplitude. This is especially an issue for configurations where the SPP given by (34) has a significantly smaller amplitude than the branch-cut contribution (35).

- A much finer minimal mesh refinement is necessary near the interface $\Sigma$ in order to resolve the highly oscillatory SPP. In addition, failure to sufficiently resolve the interface in the whole computational domain results in a suppression of the SPP due to non-local cancellation effects (pollution effect); see Section 5.2 for computational examples.

One of the major advantages of a finite element approach for discretizing variational formulation (17) is the fact that no regularization of the interface by a layer with artificial thickness is necessary. Instead, the sheet can be directly approximated as a lower-dimensional interface.

In the remainder of this section, we discuss our choice of a PML to remedy the negative effect of the absorbing boundary condition on the SPP amplitude. Further, a strategy for adaptivity and local mesh refinement is introduced, which is based on a posteriori error control combined with a fixed (a priori) local refinement of the interface.

\subsection{Perfectly matched layer}

In this subsection, we carry out a construction of a PML 42, 43, 44, for the rescaled Maxwell equations with a jump condition. The concept of a PML was pioneered by Bérenger 42. It is essentially a layer with modified material parameters $\left(\tilde{\varepsilon}_{r}, \mu_{r}\right)$ placed near the boundary such that all outgoing electromagnetic waves decay exponentially with no "artificial" reflection due to truncation of the domain. The PML is an indispensable tool for truncating unbounded domains for wave equations and often used in the numerical approximation of scattering problems [34, 45, 43, 42, 44].

We use an approach for a PML for time-harmonic Maxwell's equations outlined by Chew and Weedon [43, as well as Monk 34. The idea is to use a formal change of coordinates from the computational domain $\Omega \subset \mathbb{R}^{3}$ with realvalued coordinates to a domain $\Omega \subset\{z \in \mathbb{C}: \operatorname{Im} z \geq 0\}^{3}$ with complex-valued coordinates and non-negative imaginary part [34] and transform back to the real-valued domain. This results in modified material parameters $\tilde{\varepsilon}_{r}, \mu_{r}$ and $\sigma_{r}^{\Sigma}$ within the PML.

We assume that a PML is imposed as a concentric spherical shell in a small outer region near the boundary $\partial \Omega$; see Figure 4 . The transformation is chosen to be a function of distance $\rho$ in radial $\left(\boldsymbol{e}_{r^{-}}\right)$direction from the origin. Furthermore, assume that the normal field $\boldsymbol{\nu}$ of $\Sigma$ is orthogonal to $\boldsymbol{e}_{r}$ (for $\boldsymbol{x} \in \Sigma \cap(\mathrm{PML})$ ) and 


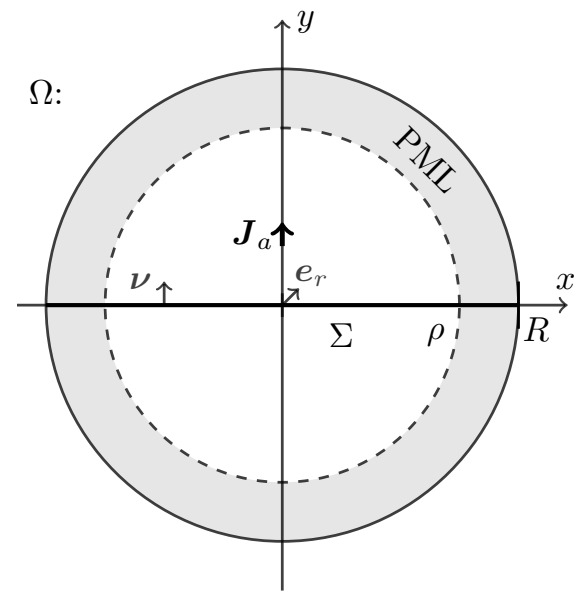

Figure 4: The computational domain $\Omega$ with a vertical dipole $\boldsymbol{J}_{a}$ situated above a planar conducting sheet $\Sigma$ located on the $x$-axis.

that $\boldsymbol{J}_{a} \equiv 0$ and $\boldsymbol{M}_{a} \equiv 0$ within the PML. Introduce a change of coordinates $\Omega \rightarrow \Omega$ with

$$
\boldsymbol{x} \mapsto \quad \dot{\boldsymbol{x}}= \begin{cases}\boldsymbol{x}+i \boldsymbol{e}_{r} \int_{\rho}^{r} s(\tau) \mathrm{d} \tau & \text { if } r \geq \rho, \\ \boldsymbol{x} & \text { otherwise }\end{cases}
$$

with $r=\boldsymbol{e}_{r} \cdot \boldsymbol{x}$ and an appropriately chosen, nonnegative scaling function $s(\tau)$ [34]. This prescription leads to a modified system of Maxwell's equations defined on $\dot{\Omega}$, which takes the following (rescaled) form within the PML:

$$
\left\{\begin{aligned}
\dot{\nabla} \times\left(\mu_{r}^{-1} \dot{\nabla} \times \dot{\boldsymbol{E}}\right)-\tilde{\varepsilon}_{r} \dot{\boldsymbol{E}} & =0, \\
{\left[\boldsymbol{\nu} \times\left(\mu_{r}^{-1} \dot{\nabla} \times \dot{\boldsymbol{E}}\right)\right]_{\Sigma} } & =i \sigma_{r}^{\Sigma} \dot{\boldsymbol{E}}_{T} \quad \text { on } \Sigma,
\end{aligned}\right.
$$

where the accent on top of $\boldsymbol{E}$ and $\nabla$ indicates the dependence on as well as the differentiation with respect to $\dot{\boldsymbol{x}}$. Next, we transform $(39)$ back from $\dot{\Omega}$ to $\Omega$ with the help of diffeomorphism (38). It follows that [34]

$$
\dot{\nabla} \times \dot{\mathcal{F}}=A(\nabla \times B \mathcal{F}), \quad \boldsymbol{\nu} \times \dot{\mathcal{F}}=C(\boldsymbol{\nu} \times B \mathcal{F}),
$$

for $\boldsymbol{\nu}$ orthogonal to $\boldsymbol{e}_{r}$. In the above, we introduce the $3 \times 3$ matrices

$$
\begin{gathered}
A=T_{\boldsymbol{e}_{x} \boldsymbol{e}_{r}}^{-1} \operatorname{diag}\left(\frac{1}{\bar{d}^{2}}, \frac{1}{d \bar{d}}, \frac{1}{d \bar{d}}\right) T_{\boldsymbol{e}_{x} \boldsymbol{e}_{r}}, \quad B=T_{\boldsymbol{e}_{x} \boldsymbol{e}_{r}}^{-1} \operatorname{diag}(d, \bar{d}, \bar{d}) T_{\boldsymbol{e}_{x} \boldsymbol{e}_{r}}, \\
C=T_{\boldsymbol{e}_{x} \boldsymbol{e}_{r}}^{-1} \operatorname{diag}\left(\frac{1}{\bar{d}}, \frac{1}{\bar{d}}, \frac{1}{d}\right) T_{\boldsymbol{e}_{x} \boldsymbol{e}_{r}}
\end{gathered}
$$

where

$$
d=1+i s(r), \quad \bar{d}=1+i / r \int_{\rho}^{r} s(\tau) \mathrm{d} \tau,
$$


and $T_{\boldsymbol{e}_{x} \boldsymbol{e}_{r}}$ is the rotation matrix that rotates $\boldsymbol{e}_{r}$ onto $\boldsymbol{e}_{x}$. Thus, applying the rescaling

$$
\left\{\begin{array}{ccc}
\mu_{r}^{-1} & \longrightarrow & \breve{\mu}_{r}^{-1}=B \mu_{r}^{-1} A, \\
\tilde{\varepsilon}_{r} & \longrightarrow & \breve{\varepsilon}_{r}=A^{-1} \tilde{\varepsilon}_{r} B^{-1} \\
\sigma_{r}^{\Sigma} & \longrightarrow & \breve{\sigma}_{r}^{\Sigma}=C^{-1} \sigma_{r}^{\Sigma} B^{-1}
\end{array}\right.
$$

to 39 leads to the system (with $\breve{\boldsymbol{E}}:=B \boldsymbol{E}$ )

$$
\left\{\begin{aligned}
\nabla \times & \left(\breve{\mu}_{r}^{-1} \nabla \times \breve{\boldsymbol{E}}\right)-\breve{\varepsilon}_{r} \breve{\boldsymbol{E}} \\
{\left[\boldsymbol{\nu} \times\left(\breve{\mu}_{r}^{-1} \nabla \times \breve{\boldsymbol{E}}\right)\right]_{\Sigma} } & =i \breve{\sigma}_{r}^{\Sigma} \breve{\boldsymbol{E}}_{T} \quad \text { on } \Sigma,
\end{aligned}\right.
$$

within the real-valued domain $\Omega$. Note that outside of the PML matrices $A, B$, and $C$ simply reduce to the unit matrix. Thus, $\boldsymbol{E}=\breve{\boldsymbol{E}}$ and (44) is identical to (11) with jump condition (15). The modified equations for the PML can be implemented by suitably replacing $\tilde{\varepsilon}_{r}, \mu_{r}^{-1}$, and $\sigma_{r}^{\Sigma}$ by their counterparts according to 43 within the PML. For the 2D model discussed in Remark 1 , the scalar $\mu_{r}^{-1}$ is transformed via

$$
\mu_{r}^{-1} \quad \longrightarrow \quad \breve{\mu}_{r}^{-1}=\frac{\mu_{r}^{-1}}{d},
$$

where $d$ is given by 42 .

\subsection{A posteriori error estimation and local refinement}

One of the computationally challenging aspects of the numerical simulation is the two-scale behavior of problem (17). A much finer minimal mesh refinement is necessary near the interface $\Sigma$ in order to resolve the highly oscillatory SPP. In this subsection we give a brief overview of a local mesh adaptation strategy based on goal-oriented a posteriori error estimation. This leads to a substantial saving in computational costs (see Section 5), due to local refinement, while maintaining an optimal convergence order in a quantity of interest 46 . We focus primarily on aspects of implementation - a full, detailed discussion is beyond the scope of this paper and will be the subject of future work.

An efficient method for a posteriori error control is the dual weighted residual (DWR) method developed by Becker and Rannacher [29, 47, 46]. This method constructs estimates of local error contributions in terms of a target functional $\mathcal{J}$ with the help of a "dual problem". More precisely, let $\mathcal{J}(\boldsymbol{E})$ be a quantity of interest given by a possibly non-linear Gâteaux-differentiable function, viz.,

$$
\mathcal{J}: \boldsymbol{H}(\operatorname{curl} ; \Omega) \rightarrow \mathbb{C} .
$$

The corresponding dual problem to $(17)$ is to find a solution $\boldsymbol{Z} \in \boldsymbol{H}(\operatorname{curl} ; \Omega)$ such that

$$
A(\boldsymbol{\varphi}, \boldsymbol{Z})=\mathrm{D}_{\boldsymbol{E}} \mathcal{J}(\boldsymbol{E})[\boldsymbol{\varphi}]
$$


for all $\boldsymbol{\varphi} \in \boldsymbol{H}(\operatorname{curl} ; \Omega)$. Here, $\mathrm{D}_{\boldsymbol{E}} \cdot[\boldsymbol{\varphi}]$ denotes the Gâteaux derivative in direction $\boldsymbol{\varphi}$ with respect to $\boldsymbol{E}$. The following result can be directly applied to variational problem (17).

Proposition 2 (Becker and Rannacher [46]). Let $\boldsymbol{E}$ and $\boldsymbol{Z}$ be the solution of (17) and (47), respectively. Let $\boldsymbol{E}_{H}$ and $\boldsymbol{Z}_{H}$ be finite element approximations of the primal and dual solution associated with a discretization $\mathbb{T}_{H}(\Omega)$ of $\Omega$. Then, up to a term $R$ of higher order:

$$
\begin{gathered}
|\mathcal{J}(\boldsymbol{E})-\mathcal{J}(\boldsymbol{U})| \leq \sum_{Q \in \mathbb{T}_{H}} \eta_{Q}+R, \quad \text { with } \\
\eta_{Q}:=\frac{1}{2}\left|\rho_{Q}\left(\boldsymbol{E}_{H}, \boldsymbol{Z}-\boldsymbol{Z}_{H}\right)+\rho_{Q}^{*}\left(\boldsymbol{Z}_{H}, \boldsymbol{E}-\boldsymbol{E}_{H}\right)\right| .
\end{gathered}
$$

Here, $\rho_{Q}$ and $\rho_{Q}^{*}$ denote the primal and dual cell-wise residual, respectively, associated with variational equations (17) and (47):

$$
\begin{aligned}
& \rho_{Q}\left(\boldsymbol{E}_{H}, \boldsymbol{Z}-\boldsymbol{Z}_{H}\right)=F\left(\left(\boldsymbol{Z}-\boldsymbol{Z}_{H}\right) \chi_{Q}\right)-A\left(\boldsymbol{E}_{H},\left(\boldsymbol{Z}-\boldsymbol{Z}_{H}\right) \chi_{Q}\right), \\
& \rho_{Q}^{*}\left(\boldsymbol{Z}_{H}, \boldsymbol{E}-\boldsymbol{E}_{H}\right)=\mathrm{D}_{\boldsymbol{E}} \mathcal{J}(\boldsymbol{E})\left[\left(\boldsymbol{E}-\boldsymbol{E}_{H}\right) \chi_{Q}\right]-A\left(\left(\boldsymbol{E}-\boldsymbol{E}_{H}\right) \chi_{Q}, \boldsymbol{E}_{H}\right),
\end{aligned}
$$

with the indicator function $\chi_{Q}$ that is 1 on the cell $Q$, and 0 otherwise. The local error indicator $\eta_{Q}$ given by 49 can now be approximated and used in a local refinement strategy [46].

Our goal is an optimal local refinement for the numerical observation of SPPs on the conducting sheet $\Sigma$. In principle, a number of choices for the quantity of interest, $\mathcal{J}(\boldsymbol{E})$, are possible in order to achieve this goal. Here, we choose the quantity

$$
\mathcal{J}(\boldsymbol{E}):=\int_{\Omega} \varpi(\boldsymbol{x})\|\nabla \times \boldsymbol{E}\|^{2} \mathrm{~d} \boldsymbol{x},
$$

with an appropriate (essentially bounded), non-negative weighting function $\varpi(\boldsymbol{x})$ that localizes the integral around the interface $\Sigma$; see Section 5 for the concrete choice of $\varpi$ for our simulations. The choice $\sqrt[52]{ }$ for the quantity of interest leads to a localized right-hand side $\mathcal{J}$ of the dual problem that is sensitive to the highly oscillatory SPP associated with the electric field, E. Consequently, the weight $\boldsymbol{Z}-\boldsymbol{Z}_{H}$ in residual 50 is generally large near the interface and at points where the influence of the solution on quantity 52 is high.

Remark 4. A purely residual-based error estimator on the other hand corresponds to a uniform weight distribution. The DWR method (with our choice of right hand side) will lead to a more localized refinement.

In order to guarantee a uniform refinement over the interface $\Sigma$, the local refinement strategy is augmented by additionally selecting all cells $Q$ for refinement that fulfill

$$
1-(1 / 2)^{\# \text { cycle }-1} \leq \varpi\left(x_{Q}\right) / \max (\varpi),
$$

where $x_{Q}$ denotes the center of $Q$. 
Remark 5 (Evaluation of residuals). A classical approach to evaluate (50) and (51) is to use a higher-order approximation for $\boldsymbol{Z}$ and $\boldsymbol{E}$ and transform $\rho_{Q}$ and $\rho_{Q}^{*}$ into a strong residual form [46]. We follow a different strategy that does not involve solving higher-order solutions. Instead of a higher-order approximation of $\boldsymbol{Z}$, we use a patch-wise projection $\pi_{2 H}^{(2)} \boldsymbol{Z}_{H}$ to a higher-order space on a coarser mesh level [48], viz.,

$$
\boldsymbol{Z}-\boldsymbol{Z}_{H} \approx \pi_{2 H}^{(2)} \boldsymbol{Z}_{H}-\boldsymbol{Z}_{H}, \quad \boldsymbol{E}-\boldsymbol{E}_{H} \approx \pi_{2 H}^{(2)} \boldsymbol{E}_{H}-\boldsymbol{E}_{H},
$$

in combination with the (variational) residuals (50) and (51).

Remark 6 (Mixed form of error estimator). Error estimator (54) also has a form with only the primal residual $\rho().($.$) appearing. However, the mixed form$ of the above error estimator should be used here to ensure adequate, simultaneous refinement not just only with respect to a localized SPP (on the interface), but also with respect to a singular right-hand side $\boldsymbol{J}_{a}$ (modeling of a Hertzian dipole).

\section{Numerical results}

In this section, we present a number of computational results pertaining to the excitation by a vertical electric dipole of an SPP on a 2D conducting sheet. First, we construct a PML which involves use of a certain parameter, $s_{0}$. Second, we demonstrate the necessity for a PML and carry out a study for the suitable choice of $s_{0}$. Third, we compare the analytical results of Section 3 to (direct) numerical simulations based on our finite element formulation of Section 4 All numerical computations are carried out with the C++ finite element toolkit deal.II [49, 14]. We use the direct solver UMFPACK [50, 51] for the resulting linear system of equations.

\subsection{Setup}

In order to carry out the numerical simulations, we consider a vertical electric dipole positioned at $\boldsymbol{a}=(0,0.75)$ and $\boldsymbol{a}=(0,1)$ above the conducting sheet. Recall that the corresponding current densities are

$$
\boldsymbol{J}_{a}=\left(\begin{array}{c}
0 \\
J_{0}
\end{array}\right) \delta(\boldsymbol{x}-\boldsymbol{a}), \quad \boldsymbol{M}_{a}=0 .
$$

The conducting sheet has surface conductivity $\sigma_{r}^{\Sigma}=\left(\sigma_{1}+i \sigma_{2}\right) I$ in tensor form $(I$ : unit second-rank tensor). Below, we carry out a parameter study with different values of constant scalar $\sigma_{r}^{\Sigma}$.

The computational domain $\Omega$ is chosen to be the ball with radius $R=8 \pi$, which is 8 times the free-space wavelength of the dipole radiation. A PML is used in the outer region at distance $\rho>0.8 R$ from the origin; see Section 4.1 . Following Monk [34, the (nonnegative) scaling function $s(\rho)$ is chosen to be

$$
s(\rho)=s_{0} \frac{(\rho-0.8 R)^{2}}{(R-0.8 R)^{2}},
$$


where $s_{0}$ is a free parameter; the suitable choice of $s_{0}$ is discussed in Section 5.2 .

We use the adaptive refinement strategy that was outlined in Section 4.2 with the quantity of interest (52) and the choice

$$
\varpi(\boldsymbol{x})= \begin{cases}\cos ^{2}\left(\frac{\pi}{2} \frac{y}{d_{\varpi}}\right), & \text { if }|y| \leq d_{\varpi}, \\ 0 & \text { otherwise. }\end{cases}
$$

The parameter $d_{\varpi}$ controls the width of the region around the interface that is uniformly refined and is chosen to be $d_{\varpi}=1.5625$, a few multiples of the SPP wavelength $2 \pi /\left(\operatorname{Re} k_{m, r}\right)$. The current density of the dipole source is regularized according to

$$
\delta(\boldsymbol{x}-\boldsymbol{a}) \approx \begin{cases}\left(\frac{\pi}{2}-\frac{2}{\pi}\right)^{-1} d^{-2} \cos ^{2}\left(\frac{\pi}{2} \frac{\|\boldsymbol{x}-\boldsymbol{a}\|}{d}\right) & \text { for }\|\boldsymbol{x}-\boldsymbol{a}\|<d, \\ 0 & \text { otherwise, }\end{cases}
$$

with $d=0.15625$. The regularization parameter $d$ should be chosen as small as possible and has to be scaled with the initial mesh size such that $\boldsymbol{J}_{a}$ is always well integrated numerically. Figure 4 summarizes the aforementioned setup.

\subsection{On perfectly matched layer}

In this subsection, we demonstrate the necessity for a PML. In our numerical setup, the challenging part of a direct finite element simulation is the two-scale character that the electromagnetic wave exhibits in the spatial resolution. Recall that the desired SPP has a wavelength much smaller than the one manifested by the dipole free-space radiation field.

In particular, we are interested in observing SPPs with an associated wave number Re $k_{m, r} \approx 10-100$, compared to the (rescaled) free-space wave number $k_{r}=1$. Moreover, the amplitude of the SPP scales exponentially with the distance, $a$, of the dipole from the interface; hence, certain configurations of physical appeal exhibit a ratio in amplitude of about 1:10 to 1:1000 between the SPPs and the dipole free-space radiation field. It turns out that absorbing boundary condition (14) that we use-although it is a first-order absorbing boundary condition-is not well suited for the numerical study of the SPP. A significant suppression of the SPP amplitude can be evident.

In order to study the influence of the absorbing boundary condition on the SPP, we perform a series of numerical simulations of the geometry given in Figure 4 for different values of $s_{0}$ which controls the absorption strength of the PML; specifically, we use $s_{0}=0,0.25,0.5,1.0,2.0,4.0$, and 8.0, respectively. The material parameters are set to $\mu_{r}=\tilde{\varepsilon}_{r}=I$. We choose $\boldsymbol{a}=(0,1)$ for the position vector of the dipole, with sheet of relatively small, purely imaginary surface conductivity, i.e.,

$$
\sigma_{r}^{\Sigma}=i \sigma_{2}=0.15 i .
$$

This value corresponds to a relatively weak SPP, i.e., with a relatively small amplitude, that has a purely real-valued wave number, $k_{m, r} \approx 13.33$. This can 


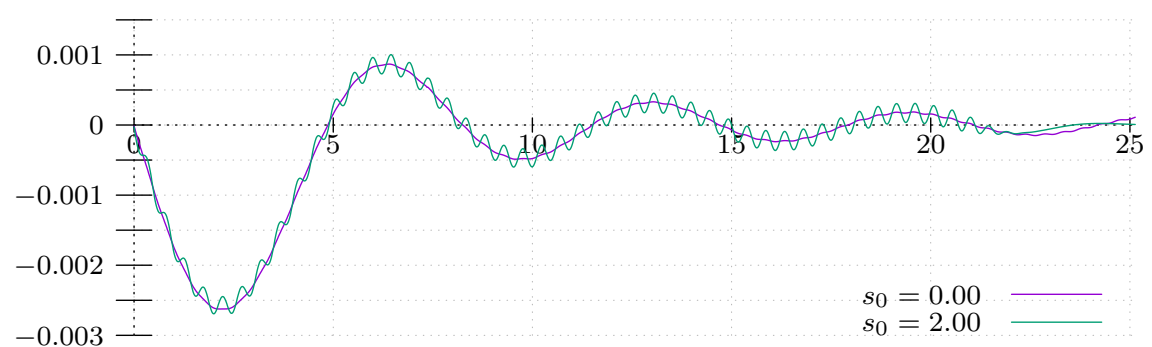

(a) Scattered $\operatorname{Re}\left(\boldsymbol{E}_{x}\right)$ as function of position $x$

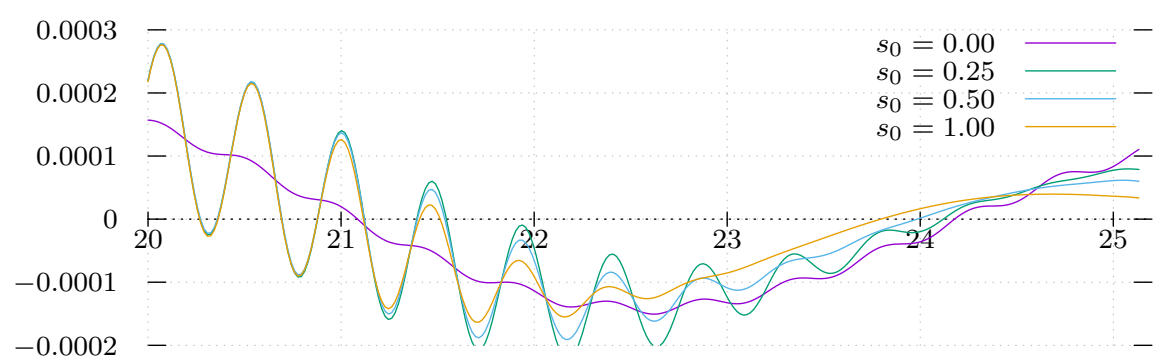

(b) zoom into the PML, $s_{0}=0.0,0.25,0.5,1.0$

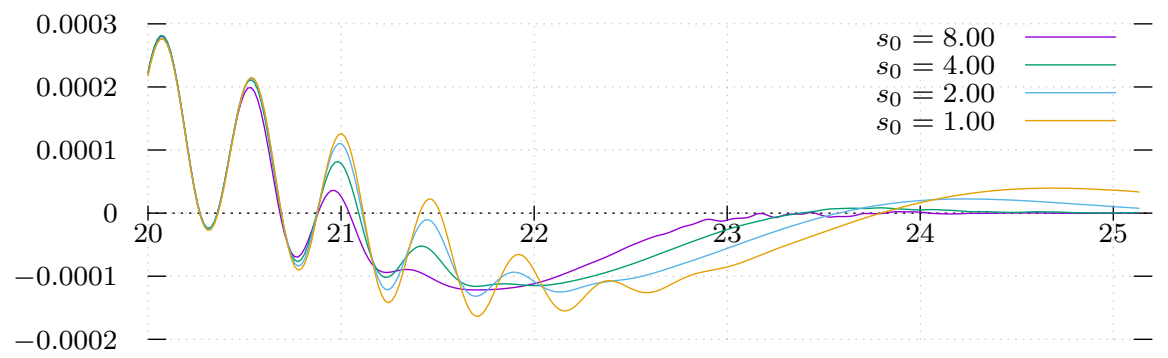

(c) zoom into the PML, $s_{0}=1.0,2.0,4.0,8.0$

Figure 5: Real part of $x$-component of scattered field, $\operatorname{Re} E_{x}^{\mathrm{sc}}=\operatorname{Re}\left(E_{x}-E_{x}^{\mathrm{pr}}\right)$, on the interface $\Sigma$ as a function of position, $x ; E_{x}^{p r}$ is the primary dipole field component, in the absence of the sheet. (a) Comparison of no PML with a PML of parameter $s_{0}=2$; (b) zoom into the PML with parameter values $s_{0}=0,0.25,0.5,1.0 ;$ (c) zoom into the PML with $s_{0}=1.0,2.0,4.0,8.0$.

be understood as follows: Because $\operatorname{Re} \sigma_{r}^{\Sigma}=0$, the sheet does not cause any dissipation and, thus, the corresponding SPP does not exhibit any decay.

In order to examine the influence of different boundary conditions and choices of parameters in some detail, we extract the $x$-component of the scattered electric field, $E_{x}^{\mathrm{sc}}=E_{x}-E_{x}^{\mathrm{pr}}$, on the interface $\Sigma$, and plot this component as a function of $x$, where $\boldsymbol{E}^{\mathrm{pr}}$ is the (primary) dipole field in the absence of the sheet; see Figure 5 . Boundary condition (14) without PML $\left(s_{0}=0\right)$ has a strong influence on the observed scattered field. While branch-cut contribution (35) of the scattered field can be observed in the numerical simulation, pole contribution (34), which 


\begin{tabular}{cc}
\hline Surface conductivity $\sigma_{r}^{\Sigma}$ & Predicted $k_{m, r}$ \\
$2.56 \times 10^{-4}+0.160 i$ & $12.5+0.02 i$ \\
$1.78 \times 10^{-4}+0.133 i$ & $15.0+0.02 i$ \\
$1.28 \times 10^{-3}+0.160 i$ & $12.5+0.1 i$ \\
$8.89 \times 10^{-4}+0.133 i$ & $15.0+0.1 i$ \\
\hline
\end{tabular}

Table 1: Values of $\sigma_{r}^{\Sigma}$ used in the parameter study along with the predicted SPP wave numbers $k_{m, r}$ by 60 .

is responsible for the fast oscillation with wave number $k_{m, r}$, is suppressed (Figure $5 \mathrm{a}$ ). This property can be explained by the fact that boundary condition (14), viz.,

$$
\boldsymbol{n} \times \boldsymbol{B}+\sqrt{\mu_{r}^{-1} \tilde{\varepsilon}_{r}} \boldsymbol{E}_{T}=0,
$$

is only applicable to dipole radiation with a wave number $k_{r}=1$. In the case where the pole contribution is characterized, e.g., by $k_{m, r} \approx 13.3$, this boundary condition causes a reflection that results in a suppression of the fast spatial oscillation of the SPP. In light of the parameter study (Fig. 5), we choose a PML with strength $s_{0}=2$ for all subsequent computations. This is a balanced choice between a PML that is strong enough to minimize the influence of the boundary $\left(s_{0} \geq 2\right)$ and the unwanted influence of the PML $\left(s_{0} \leq 2\right)$; cf. Figure 5 .

\subsection{Comparison of numerical results to analytical solution}

In this subsection, we compare our numerical results to the analytical solution of Section 3 In particular, we expect to observe the SPP described by (34). The (complex-valued) wave number $k_{m, r}$ associated with this SPP scales with the surface conductivity $\sigma_{r}^{\Sigma}$ as follows (given in rescaled quantities, as explained in Section 2.2):

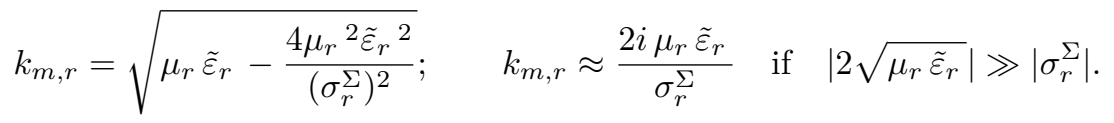

In order to test our numerical method (Section 4) against the analytical results (Section 3), we carry out a parameter study for a variety of different values of $\sigma_{r}^{\Sigma}$; see Table 1

Our computations are performed for dipole elevation distances $a=0.75,1.00$. For each choice of parameters, we start with a relatively coarse mesh (with 10k degrees of freedom) for the numerical simulation and run 6 mesh-adaptation cycles (with approximately $1.6 \mathrm{M}$ degrees of freedom on the finest mesh.) Figure 6 shows the $x$-directed scattered field for $\sigma_{r}^{\Sigma}=4.0 \times 10^{-4}+0.2 i\left(k_{m} \approx 10.0+0.02 i\right)$, and $\sigma_{r}^{\Sigma}=2.0 \times 10^{-3}+0.2 i\left(k_{m} \approx 10.0+0.1 i\right)$. For this choice of parameters, a relatively strong pole contribution can be observed as shown in Figure 6. (b). Figure 7 shows the locally refined mesh after the final refinement cycle.

For a comparison of our numerical method to the analytical results of Section 3 the pole contribution (34) and branch-cut contribution (35) are computed 


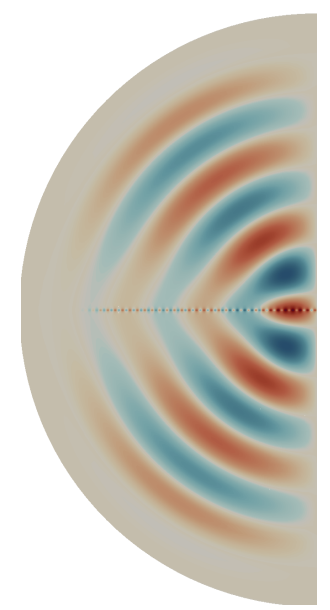

(a) weak absorption

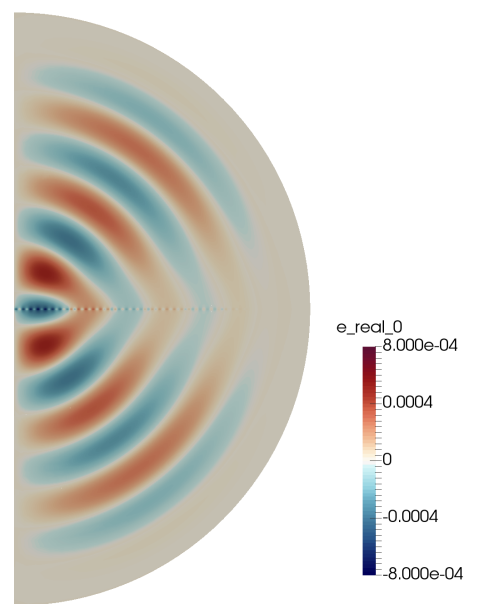

(b) strong absorption

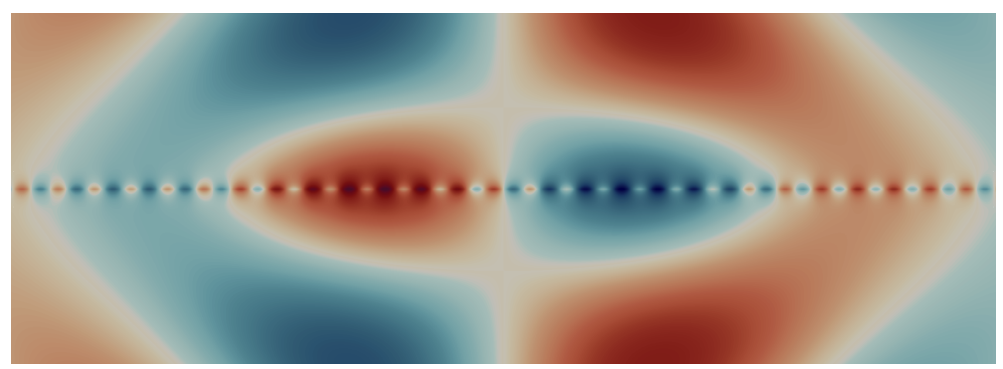

(c) Zoom of (b) around origin with detailed SPP

Figure 6: Real part of $x$-directed scattered electric field, $\operatorname{Re}\left(E_{x}^{\mathrm{sc}}\right)$, on interface $\Sigma$, computed for: (a) $\sigma_{r}^{\Sigma}=4.0 \times 10^{-4}+0.2 i\left(k_{m} \approx 10.0+0.02 i\right)$; and (b) $\sigma_{r}^{\Sigma}=2.0 \times 10^{-3}+0.2 i\left(k_{m} \approx 10.0+0.1 i\right)$. The dipole elevation distance is $a=1.00$.

numerically. For this purpose, we use a summed trapezoidal rule to evaluate the two integrals involved in the branch-cut contribution. For the improper integral over $(0, \infty)$, we further exploit an exponential decay of the integrand (as a function of the integration variable, $\varsigma$ ) and introduce a cutoff at $\varsigma \approx 1 / \sqrt{h x}$, where $h$ is the interval size of the summed trapezoidal rule. This $h$ is chosen adaptively in an iterative cycle such that the relative change in the value of the integral between $2 h$ and $h$ is less than $0.5 \%$.

In Figures 8 and 9, the analytical and numerical results are compared graphically for dipole elevation distances $a=0.75,1.00$ and $k_{m, r}=12.5+$ $0.02 i, 15.0+0.1 i$. The real and imaginary parts of the scattered electric field in the $x$-direction, $E_{x}^{\mathrm{sc}}$, are plotted as a function of position $x$ on the interface, $\Sigma$. It is evident that the numerical and analytical results are in excellent agreement outside the PML $(0 \leq x \leq 20)$.

Table 2 summarizes the parameter study quantitatively. The $L^{2}$-error between 


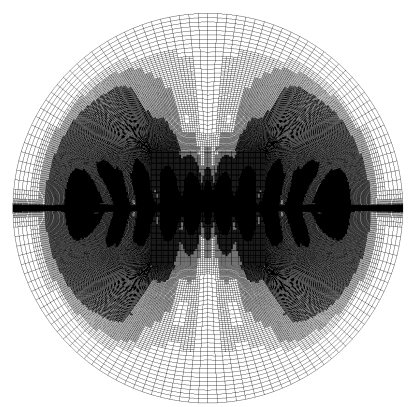

(a) full mesh

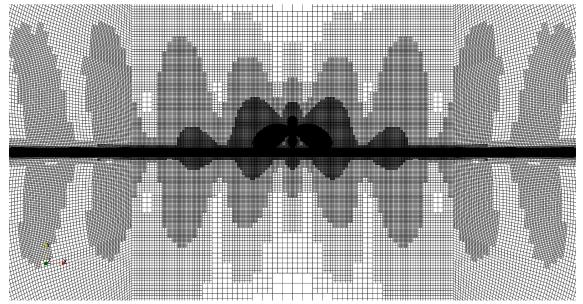

(b) zoom

Figure 7: The locally refined mesh (a) obtained with the adaptive method outlined in Section 4.2 The mesh has a total number of around 200 thousand cells; the finest resolution around the interface corresponds to a uniformly refined mesh of around 5 million cells.

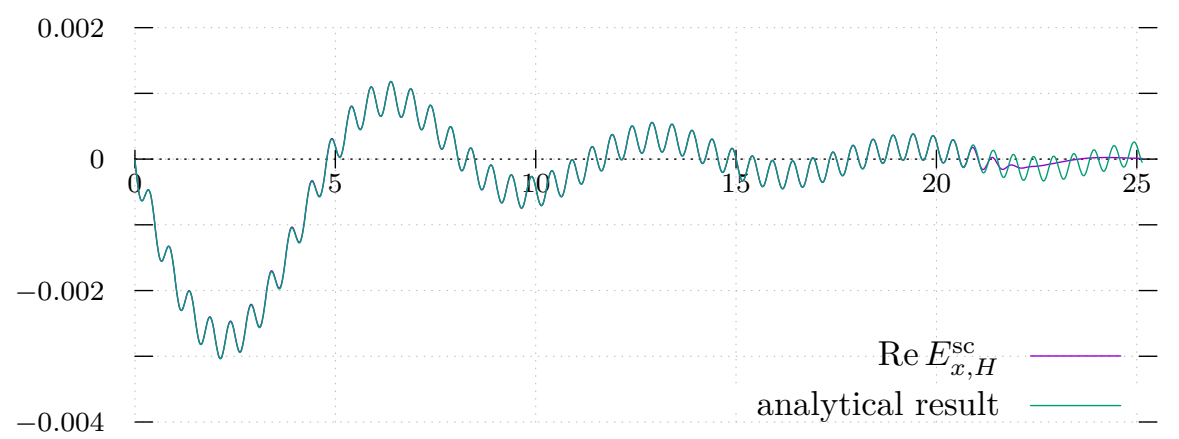

(a)

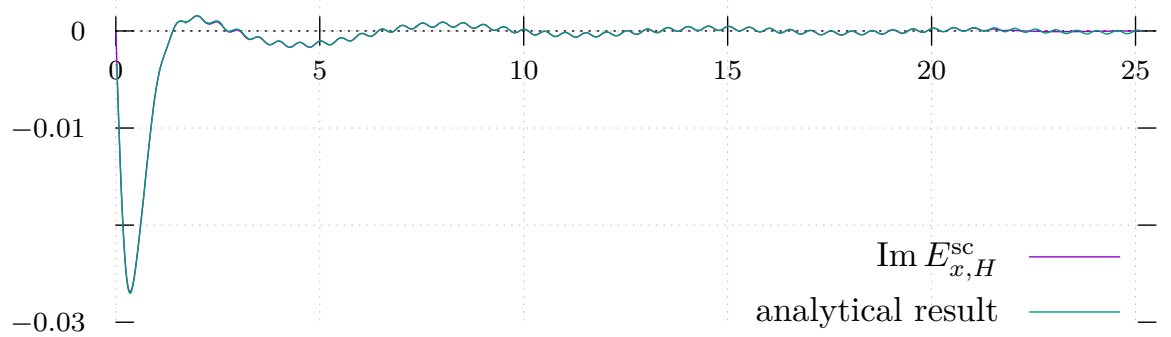

(b)

Figure 8: Real part (a) and imaginary part (b) of the $x$-component of scattered electric field, $E_{x}^{\mathrm{sc}}$, as a function of position $x$ on interface $\Sigma$, in the presence of a vertical electric dipole at distance $a$ from the conducting sheet. The plots (a-b) show the numerical simulations based on our method as well as the analytical results computed by 34 and 35 for the values $a=1.00$, $k_{m, r}=12.5+0.02 i$. 


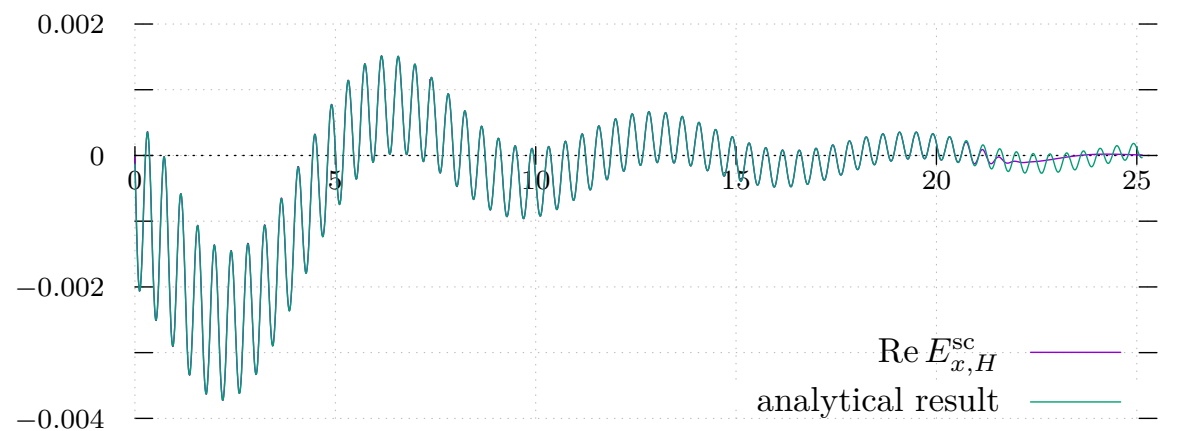

(a)

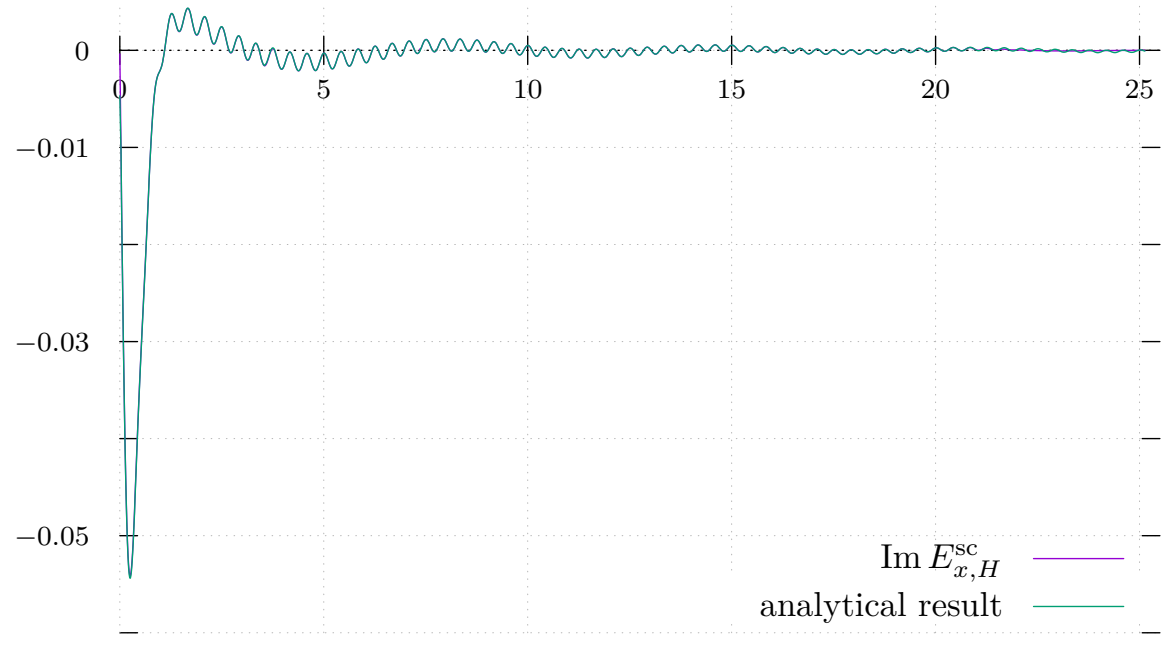

(b)

Figure 9: Real part (a) and imaginary part (b) of the $x$-component of scattered electric field, $E_{x}^{\mathrm{sc}}$, as a function of position $x$ on interface $\Sigma$, in the presence of a vertical electric dipole at distance $a$ from the conducting sheet. The plots (a-b) show the numerical simulations based on our method as well as the analytical results computed by (34) and 35 for the values $a=0.75$, $k_{m, r}=15.0+0.1 i$. 
numerical and analytical result is computed outside of the PML on the interface $\Sigma$. The SPP is generally well approximated after the 4 th cycle with a resolution of around 100 thousand degrees of freedom. According to (37), we expect a convergence order of $\left\|\boldsymbol{E}_{h, T}-\boldsymbol{E}_{T}\right\|_{L^{2}(\Omega)} \sim \mathcal{O}$ (\#dofs). Indeed, we observe a linear convergence of the error with respect to the number of refinement steps, and thus, a linear convergence in number of degrees of freedom.

\section{Conclusion and outlook}

In this paper, we developed a variational framework for the numerical simulation of electromagnetic SPPs excited by current-carrying sources along an infinitely thin conducting sheet, e.g., single-layer graphene. The sheet is modeled by an idealized, oriented hypersurface. The effect of the induced surface current of the sheet was taken into account in the corresponding boundary value problem of Maxwell's equations via jump condition (3) for the tangential component of the magnetic field; this jump is proportional to the surface current density. The conductivity of the sheet is a parameter that controls the strength of this discontinuity.

One of the main advantages offered by our approach is the natural incorporation of the jump condition in a variational formulation as a weak discontinuity, without regularization of the interface by a layer of finite thickness. We tested our numerical treatment against analytical predictions in the case with a vertical dipole radiating over an infinite conducting sheet in $2 \mathrm{D}$, and observed excellent agreement. In our numerical treatment, a linear asymptotic reduction rate could be observed for all testcases. Notably, the use of an adaptive local refinement procedure within our approach achieved significant economy in the total number of degrees of freedom in comparison to uniform mesh refinement.

Our numerics admit several generalizations and extensions. For instance, the treatment of the jump condition as a weak discontinuity over an interface is not limited to a simple (lower-dimensional) hyperplane; it can be generalized to reasonably arbitrary hypersurfaces. In fact, technically speaking, our variational framework can be readily used without modifications to model any geometry that is meshable by quadrilaterals. This flexibility should enable efficient numerical simulations of complex geometries, e.g., waveguides that contain a few graphene layers [16. Although our numerical results focused on 2D thus far, our underlying choice of local adaptivity can lead to a significant reduction of computational cost in higher spatial dimension.

\section{References}

[1] A. H. C. Neto, F. Guinea, N. M. R. Peres, K. S. Novoselov, A. K. Geim, The electronic properties of graphene, Reviews of Modern Physics 81 (2009) 109-162.

[2] D. Pesin, A. H. MacDonald, Spintronics and pseudospintronics in graphene and topological insulators, Nature Materials 11 (2012) 419-425. 


\begin{tabular}{|c|c|c|c|c|c|c|c|c|}
\hline \multirow[b]{2}{*}{ Cycle } & \multicolumn{4}{|c|}{$k_{m, r} \approx 12.5+0.02 i$} & \multicolumn{4}{|c|}{$k_{m, r} \approx 12.5+0.1 i$} \\
\hline & Cells & DoFs & $L^{2}$-error & & Cells & DoFs & $L^{2}$-error & \\
\hline 1 & 1280 & 10304 & $2.49 \mathrm{e}-2$ & - & 1280 & 10304 & $1.52 \mathrm{e}-2$ & - \\
\hline 2 & 2624 & 21660 & $7.08 \mathrm{e}-1$ & -4.83 & 2624 & 21660 & $2.03 \mathrm{e}+0$ & -7.06 \\
\hline 3 & 5474 & 45580 & $2.48 \mathrm{e}-2$ & 4.84 & 5450 & 45388 & $1.41 \mathrm{e}-2$ & 7.17 \\
\hline 4 & 11930 & 99020 & $1.41 \mathrm{e}-2$ & 0.82 & 11882 & 98636 & $8.09 \mathrm{e}-3$ & 0.80 \\
\hline 5 & 27908 & 229872 & $7.00 \mathrm{e}-3$ & 1.01 & 27842 & 229344 & $4.03 \mathrm{e}-3$ & 1.00 \\
\hline 6 & 71246 & 582828 & $3.60 \mathrm{e}-3$ & 0.96 & 71114 & 581796 & $2.03 \mathrm{e}-3$ & 0.99 \\
\hline 7 & 191906 & 1561336 & $1.81 \mathrm{e}-3$ & 0.99 & 191672 & 1559404 & $1.02 \mathrm{e}-3$ & 1.00 \\
\hline
\end{tabular}

(a) $a=0.75, k_{m} \approx 12.5+0.02 i, k_{m} \approx 12.5+0.1 i$

\begin{tabular}{|c|c|c|c|c|c|c|c|c|}
\hline \multirow[b]{2}{*}{ Cycle } & \multicolumn{4}{|c|}{$k_{m, r} \approx 15.0+0.02 i$} & \multicolumn{4}{|c|}{$k_{m, r} \approx 15.0+0.1 i$} \\
\hline & Cells & DoFs & $L^{2}$-error & & Cells & DoFs & $L^{2}$-error & \\
\hline 1 & 1280 & 10304 & $5.33 \mathrm{e}-3$ & - & 1280 & 10304 & $4.35 \mathrm{e}-3$ & - \\
\hline 2 & 2624 & 21660 & $4.94 \mathrm{e}-3$ & 0.11 & 2624 & 21660 & $1.24 \mathrm{e}-2$ & -1.51 \\
\hline 3 & 5474 & 45556 & $9.10 \mathrm{e}-3$ & -0.88 & 5474 & 45556 & $4.40 \mathrm{e}-3$ & 1.50 \\
\hline 4 & 11906 & 98828 & $4.17 \mathrm{e}-3$ & 1.13 & 11906 & 98828 & $2.18 \mathrm{e}-3$ & 1.01 \\
\hline 5 & 27890 & 229704 & $1.78 \mathrm{e}-3$ & 1.23 & 27890 & 229704 & $1.04 \mathrm{e}-3$ & 1.06 \\
\hline 6 & 71228 & 582684 & $9.28 \mathrm{e}-4$ & 0.94 & 71228 & 582672 & $5.27 \mathrm{e}-4$ & 0.99 \\
\hline 7 & 191978 & 1561852 & $4.69 \mathrm{e}-4$ & 0.99 & 191978 & 1561852 & $2.64 \mathrm{e}-4$ & 1.00 \\
\hline
\end{tabular}

(b) $a=0.75, k_{m} \approx 15.0+0.02 i, k_{m} \approx 15.0+0.1 i$

\begin{tabular}{|c|c|c|c|c|c|c|c|c|}
\hline \multirow[b]{2}{*}{ Cycle } & \multicolumn{4}{|c|}{$k_{m, r} \approx 12.5+0.02 i$} & \multicolumn{4}{|c|}{$k_{m, r} \approx 12.5+0.1 i$} \\
\hline & Cells & DoFs & $L^{2}$-error & & Cells & DoFs & $L^{2}$-error & \\
\hline 1 & 1280 & 10304 & $2.96 \mathrm{e}-3$ & - & 1280 & 10304 & $2.81 \mathrm{e}-3$ & - \\
\hline 2 & 2600 & 21504 & $1.37 \mathrm{e}-1$ & -5.54 & 2600 & 21504 & $3.94 \mathrm{e}-1$ & -7.13 \\
\hline 3 & 5402 & 44980 & $1.33 \mathrm{e}-3$ & 6.69 & 5402 & 44980 & $8.96 \mathrm{e}-4$ & 8.78 \\
\hline 4 & 11786 & 97868 & $6.39 \mathrm{e}-4$ & 1.06 & 11786 & 97868 & $4.02 \mathrm{e}-4$ & 1.16 \\
\hline 5 & 27728 & 228240 & $3.26 \mathrm{e}-4$ & 0.97 & 27728 & 228240 & $2.06 \mathrm{e}-4$ & 0.96 \\
\hline 6 & 71366 & 584010 & $1.68 \mathrm{e}-4$ & 0.96 & 71366 & 583998 & $1.04 \mathrm{e}-4$ & 0.99 \\
\hline 7 & 194786 & 1584772 & $8.74 \mathrm{e}-5$ & 0.94 & 194786 & 1584760 & $5.91 \mathrm{e}-5$ & 0.82 \\
\hline
\end{tabular}

(c) $a=1.00, k_{m} \approx 12.5+0.02 i, k_{m} \approx 12.5+0.1 i$

\begin{tabular}{|c|c|c|c|c|c|c|c|c|}
\hline \multirow[b]{2}{*}{ Cycle } & \multicolumn{4}{|c|}{$k_{m, r} \approx 15.0+0.02 i$} & \multicolumn{4}{|c|}{$k_{m, r} \approx 15.0+0.1 i$} \\
\hline & Cells & DoFs & $L^{2}$-error & & Cells & DoFs & $L^{2}$-error & \\
\hline 1 & 1280 & 10304 & $2.30 \mathrm{e}-3$ & - & 1280 & 10304 & $2.20 \mathrm{e}-3$ & - \\
\hline 2 & 2600 & 21504 & $6.43 \mathrm{e}-4$ & 1.84 & 2600 & 21504 & $2.20 \mathrm{e}-3$ & 0.00 \\
\hline 3 & 5426 & 45196 & $6.24 \mathrm{e}-4$ & 0.04 & 5426 & 45196 & $4.38 \mathrm{e}-4$ & 2.32 \\
\hline 4 & 11834 & 98276 & $1.84 \mathrm{e}-4$ & 1.76 & 11834 & 98276 & $1.66 \mathrm{e}-4$ & 1.40 \\
\hline 5 & 27812 & 228876 & $9.29 \mathrm{e}-5$ & 0.98 & 27812 & 228876 & $8.67 \mathrm{e}-5$ & 0.94 \\
\hline 6 & 71546 & 585474 & $4.96 \mathrm{e}-5$ & 0.90 & 71546 & 585474 & $4.60 \mathrm{e}-5$ & 0.91 \\
\hline 7 & 195095 & 1587232 & $3.30 \mathrm{e}-5$ & 0.59 & 195095 & 1587232 & $3.15 \mathrm{e}-5$ & 0.55 \\
\hline
\end{tabular}

(d) $a=1.00, k_{m} \approx 15.0+0.02 i, k_{m} \approx 15.0+0.1 i$

Table 2: Convergence history and $L^{2}$ error of the real part of the scattered electric field in $x$-direction between the numerical and analytical solution computed outside of the PML on the interface $\Sigma$. The columns after the $L^{2}$ error are the $\log _{2}$ reduction rates of the error. 
[3] B. Trauzettel, D. V. Bulaev, D. Loss, G. Burkard, Spin qubits in graphene quantum dots, Nature Physics 3 (2007) 192-196.

[4] M. A. Green, S. Pillai, Harnessing plasmonics for solar cells, Nature Photonics 6 (2012) 130-132.

[5] A. K. Geim, I. V. Grigorieva, Van der Waals heterostructures, Nature 499 (2013) 419-425.

[6] L. E. F. F. Torres, S. Roche, J.-C. Charlier, Introduction to GrapheneBased Nanomaterials: From Electronic Structure to Quantum Transport, Cambridge University Press, Cambridge, UK, 2014.

[7] X. C. Zhang, J. Xu, Introduction to THz Wave Photonics, Springer, Berlin, 2010.

[8] T. Low, R. Roldán, H. Wang, F. Xia, P. Avouris, L. M. Moreno, F. Guinea, Plasmons and screening in monolayer and multilayer black phosphorus, Phys. Rev. Lett. 113 (2014) 106802.

[9] J. M. Pitarke, V. M. Silkin, E. V. Chulkov, P. M. Echenique, Theory of surface plasmons and surface-plasmon polaritons, Reports on Progress in Physics 70 (2007) 1-87.

[10] Y. V. Bludov, A. Ferreira, N. Peres, M. I. Vasileskiy, A primer on surface plasmon-polaritons in graphene, International Journal of Modern Physics 27 (10) (2013) 1341001.

[11] P. Alonso-González, A. Y. Nikitin, F. Golmar, A. Centeno, A. Pesquera, S. Vélez, J. Chen, G. Navickaite, F. Koppens, A. Zurutuza, F. Casanova, L. E. Hueso, R. Hillenbrand, Controlling graphene plasmons with resonant metal antennas and spatial conductivity patterns, Science 344 (2014) 13691373 .

[12] Y. Liu, S. Palomba, Y. Park, T. Zentgraf, X. Yin, X. Zhang, Compact magnetic antennas for directional excitation of surface plasmons, Nano Letters 12 (2012) 4853-4858.

[13] J. Cheng, W. L. Wang, H. Mosallaei, E. Kaxiras, Surface plasmon engineering in graphene functionalized with organic molecules: A multiscale theoretical investigation, Nano Letters 14 (2014) 50-56.

[14] W. Bangerth, T. Heister, L. Heltai, G. Kanschat, M. Kronbichler, M. Maier, B. Turcksin, The deal. II Library, Version 8.3, submitted.

[15] B. Gallinet, J. Butet, O. J. F. Martin, Numerical methods for nanophotonics: standard problems and future challenges, Laser and Photonics Reviews 9 (2015) 577-603.

[16] C. Yeh, F. Shimabukuro, The Essence of Dielectric Waveguides, Springer, New York, 2008. 
[17] F. H. L. Koppens, D. E. Chang, F. J. G. de Abajo, Graphene plasmonics: A platform for strong light-matter interactions, Nano Letters 11 (2011) $3370-3377$.

[18] Yuan-Fong, H.-H. Yeh, A comparative study of solid-silver and silver-shell nanodimers on surface plasmon resonances, Journal of Nanoparticle Research 13 (2011) 637-644.

[19] J. B. Edel, A. A. Kornyshev, A. R. Kucernaka, M. Urbakh, Fundamentals and applications of self-assembled plasmonic nanoparticles at interfaces, Chemical Society Reviews 45 (2016) 1581-1596.

[20] D. Margetis, M. Luskin, On solutions of Maxwell's equations with dipole sources over a thin conducting film, Journal of Mathematical Physics 57 (2016) 042903.

[21] H. Raether, Surface Plasmons on Smooth and Rough Surfaces and on Gratings, Springer, Berlin, 1986.

[22] R. W. P. King, M. Owens, T. T. Wu, Lateral Electromagnetic Waves: Theory and Applications to Communications, Geophysical Exploration, and Remote Sensing, Springer-Verlag, New York, 1992.

[23] C. Müller, Foundations of the Mathematical Theory of Electromagnetic Waves, Springer-Verlag, New York, 1969.

[24] M. Schwartz, Principles of Electrodynamics, International Series in Pure and Applied Physics, McGraw-Hill Book Company, New York, 1972.

[25] S. A. Maier, Plasmonics: Fundamentals and Applications, Springer, New York, 2007.

[26] N. Dewapriya, N. Rajapake, N. Nigam, Influence of hydrogen functionalization on the fracture strength of graphene and the interfacial properties of graphene-polymer nanocomposite, Carbon 93 (2015) 830-842.

[27] G. W. Hanson, Dyadic Green's functions and guided surface waves for a surface conductivity model of graphene, Journal of Applied Physics 103 (6) (2008) 064302, erratum, ibid. 113(2):029902, 2013.

[28] A. Satou, S. A. Mikhailov, Excitation of two-dimensional plasmon polaritons by an incident electromagnetic wave at a contact, Phys. Rev. B 75 (2007) 045328 .

[29] R. Becker, R. Rannacher, A Feed-Back Approach to Error Control in Finite Element Methods: Basic Analysis and Examples, East-West Journal of Numerical Mathematics 4 (1996) 237-264.

[30] G. W. Hanson, A. B. Yakovlev, A. Mafi, Excitation of discrete and continuous spectrum for a surface conductivity model of graphene, Journal of Applied Physics 110 (11) (2011) 114305. 
[31] A. Y. Nikitin, F. Guinea, F. J. Garcia-Vidal, L. Martin-Moreno, Fields radiated by a nanoemitter in a graphene sheet, Phys. Rev. B 84 (2011) 195446.

[32] S. C. Brenner, J. Gedicke, L.-Y. Sung, An adaptive $P_{1}$ finite element method for two-dimensional Maxwell's equations, Journal of Scientific Computing 55 (3) (2013) 738-754.

[33] S. C. Brenner, J. Gedicke, L.-Y. Sung, An adaptive $P_{1}$ finite element method for two-dimensional transverse magnetic time harmonic Maxwell's equations with general material properties and general boundary conditions, Journal of Scientific Computing 68 (2) (2016) 848-863.

[34] P. Monk, Finite Element Methods for Maxwell's Equations, Numerical Mathematics and Scientific Computation, Oxford University Press, 2003.

[35] J.-C. Nédélec, A new family of mixed finite elements in $\mathbb{R}^{3}$, Numerische Mathematik 50 (1986) 57-81.

[36] J.-C. Nédélec, Acoustic and Electromagnetics Equations, Vol. 144 of Applied Mathematical Sciences, Springer, 2001.

[37] A.-S. Bonnet-Ben Dhia, L. Chesnel, P. Ciarlet Jr., T-coercivity for the Maxwell problem with sign-changing coefficients, Communications in Partial Differential Equations 39 (2014) 1007-1031.

[38] D. Colton, R. Kress, Integral Equation Methods in Scattering Theory, Vol. 72 of Classics in Applied Mathematics, SIAM, 2013.

[39] J.-M. Jin, D. J. Riley, Finite Element Analysis of Antennas and Arrays, Wiley, 2009.

[40] D. Colton, R. Kress, Inverse Acoustic and Electromagnetic Scattering Theory, 3rd Edition, Applied Mathematical Sciences, Springer, 2013.

[41] V. Bokil, N. Gibson, V. Gyrya, D. McGregor, Dispersion reducing methods for edge discretizations of the electric vector wave equation, Journal of Computational Physics 287 (2015) $88-109$.

[42] J.-P. Bérenger, A perfectly matched layer for the absorption of electromagnetic waves, Journal of Computational Physics 114 (1994) 185-200.

[43] W. C. Chew, W. H. Weedon, A 3D perfectly matched medium from modified Maxwell's equations with stretched coordinates, Microwave and Optical Technology Letters 7 (13) (1994) 599-604.

[44] L. Zhao, A. C. Cangellaris, A general approach for the development of unsplit-field time-domain implementations of perfectly matched layers for FDTD grid truncation, IEEE Microwave and Guided Wave Letters 6 (5) (1996) 209-211. 
[45] G. Bao, P. Li, H. Wu, An adaptive edge element method with perfectly matched absorbing layers for wave scattering by biperiodic structures, Mathematics of Computation 79 (2010) 1-34.

[46] R. Becker, R. Rannacher, An Optimal Control Approach to A Posteriori Error Estimation in Finite Element Methods, Acta Numerica 10 (2001) $1-102$.

[47] R. Becker, R. Rannacher, Weighted A Posteriori Error Control in FE Methods, in: Proceedings of ENUMATH-97, 1998, pp. 621-637, lecture at ENUMATH-95, Paris, September 18-22, 1995.

[48] M. Braack, T. Richter, Solutions of 3D Navier-Stokes Benchmark Problems With Adaptive Finite Elements, Computers and Fluids 35 (2006) 27-392.

[49] W. Bangerth, T. Heister, L. Heltai, G. Kanschat, M. Kronbichler, M. Maier, B. Turcksin, T. D. Young, The deal. II Library, Version 8.2, Archive of Numerical Software 3.

[50] T. A. Davis, Algorithm 915, SuiteSparseQR: Multifrontal Multithreaded Rank-Revealing Sparse QR Factorization (2011).

[51] T. A. Davis, P. R. Amestoy, I. S. Duff, et al., SuiteSparse 4.2.1, A Suite of Sparse Matrix Software (2013). 